\title{
Aktuelle Entwicklungen an den österreichischen
}

\section{Bibliotheken 2017 bis 2018}

Zusammenfassung: Im vorliegenden Beitrag wird über aktuelle Projekte und Trends im österreichischen Bibliothekswesen in den Jahren 2017 und 2018 berichtet. Vorgestellt werden Verbundprojekte und Kooperationsprojekte österreichischer Bibliotheken, aktuelle Projekte der Österreichischen Nationalbibliothek und des Forums Universitätsbibliotheken Österreichs. Angesprochen werden auch Themen wie Forschungsdaten, Open Access, NS-Provenienzforschung, Bibliotheksausbildung, Tagungen und Publikationen der Vereinigung Österreichischer Bibliothekarinnen und Bibliothekare sowie Bibliotheks- und Informationspolitik in Österreich.

Schlüsselwörter: Österreich; Bibliothekswesen; Entwicklung; 2017; 2018

\section{Current Developments in Austrian Libraries in 2017 and2018}

Abstract: The present contribution relates about current projects and trends in Austrian librarianship in 2017 and 2018. Network projects and cooperation projects are presented as well as important projects of the Austrian National Library and of the Council of Austrian University Libraries. Themes such as Open Access, research data, provenance research of Nazilooted property, library education, conferences and publications of the Austrian Association of Librarians and library and information policy in Austria are mentioned.

Keywords: Austria; librarianship; development; 2017; 2018

\section{1 Österreichischer Bibliothekenverbund}

\subsection{Neues Bibliothekssystem Alma}

Erste Überlegungen für die Ablösung des bisher im Österreichischen Bibliothekenverbund eingesetzten Bibliothekssystems Aleph 500 wurden bereits 2009 angestellt. Danach erfolgten eine Marktsondierung sowie die Erstellung eines Pflichtenheftes. 2013 bis 2015 wurde ein Verhandlungsverfahren von 14 Auftraggeberinstitutionen durchgeführt, welches im September 2015 mit dem Zuschlag für das Produkt ALMA der Bestbieterfirma Ex Libris erfolgreich beendet wurde. Nach einer vorangegangenen sechsmonatigen Konzeptionsphase erfolgten zwischen August 2016 
und März 2018 die Implementierung sowie die Inbetriebnahme des neuen Systems an der Österreichischen Bibliothekenverbund und Service Gesellschaft m.b.H. (OBVSG) und den 13 am Verfahren beteiligten Bibliotheken, die in zwei Kohorten organisiert waren.

Die weitere Migration des Verbundes ist in mehreren Schritten bis Sommer 2021 geplant, wobei die jeweils involvieren Einrichtungen in sieben Waves gegliedert werden; bereits im September 2018 konnte die Implementierung an drei weiteren Verbundbibliotheken erfolgreich abgeschlossen werden. ${ }^{1}$ Eine besonders wichtige Funktion bei Vorbereitung und Umsetzung des Systemwechsels kam den Expertinnen und Experten an den einzelnen Bibliotheken zu; diese arbeiteten in acht Gruppen - vom Bereich der Daten über E-Ressourcen, Entlehnung, Erwerbung, Fernleihe, Katalogisierung bis zu Öffentliche Services und Zeitschriften - sowie einer Schulungsgruppe und fungierten als Schnittstelle zur OBVSG und zur Herstellerfirma. ${ }^{2}$ Die Herausforderungen sowie die einzelnen Arbeitsschritte des Umstiegs wurden in Erfahrungsberichten der Bibliothek der Fachhochschule Sankt Pölten ${ }^{3}$ sowie der Universitätsbibliothek $\mathrm{Graz}^{4}$ festgehalten.

Der Umstieg bringt auch gravierende Änderungen für die konkrete Formal- und SachErschließungsarbeit mit sich, insbesondere auch, weil im Österreichischen Bibliothekenverbund nunmehr von den ALMA-Bibliotheken MARC21 anstelle von MAB2 als Katalogisierungsformat genutzt wird. ${ }^{5}$ Aufgrund der mehrjährigen Übergangsphase von Aleph 500 zu ALMA im Österreichischen Bibliothekenverbund ist ein entsprechend langer Parallelbetrieb in der Verbundarchitektur erforderlich. Weiters mussten verschiedene zentrale Services, wie Primo, GND, Visual Library, IV-Scan und SAP-Anbindung, entsprechend den neuen Anforderungen angepasst werden. ${ }^{6}$ Auch der bisher eigenständig betriebene Katalog für Nachlässe, Autografen und Handschriften wurde in die neue Verbunddatenbank migriert, wobei auch eine Umstellung der Datenbestände auf das neue Datenformat MARC21 erfolgte. ${ }^{7}$

\subsection{Primo}

Mit dem Systemwechsel von Aleph zu ALMA erfolgten auch bei der an vielen Verbundbibliotheken eingesetzten Suchmaschine PRIMO zahlreiche Anpassungen, was mit der Etablierung einer

\footnotetext{
${ }^{1}$ Kronschläger und Mathis (2018a), Kronschläger und Mathis (2018b) und OBV (2018a).

2 Bauer et al. (2018b).

${ }^{3}$ Kieslinger und Rathmanner (2018).

${ }^{4}$ Lackner (2018).

${ }^{5}$ Beiler et al. (2018).

${ }^{6}$ Kann (2017) und Kann (2018).

7 OBV (2017f).
} 
Primo4Alma-Instanz ermöglicht wurde. ${ }^{8}$ Parallel zur Inbetriebnahme der ALMA-Netzwerkzone im März 2018 erfolgte auch in der Verbundsuchmaschine die Umstellung auf MARC21. ${ }^{9}$

In der Verbundsicht von PRIMO gibt es neben dem Reiter Fachliteratur noch den Sucheinstieg für Hochschulschriften, der eine gezielte Recherche nach Bachelorarbeiten, Masterarbeiten, Diplomarbeiten, Dissertationen und Habilitationsschriften ermöglicht, ${ }^{10}$ und den Sucheinstieg für Nachlässe \& Handschriften. ${ }^{11}$ Die jeweils zugeordneten Datensätze werden bereits bei der Katalogisierung entsprechend gekennzeichnet, sodass eine exakte Recherche nach den jeweiligen Kategorien durchgeführt werden kann.

\subsection{Visual Library}

Bis Ende 2018 ist die Zahl der Anwenderbibliotheken von Visual Library um weitere drei Institutionen ${ }^{12}$ auf zwölf Bibliotheken (neun Universitätsbibliotheken und drei Fachhochschulbibliotheken) angestiegen.

Ein besonders attraktiver Bestand wurde von der Bibliotheca Mozartiana der Stiftung Mozarteum Salzburg in ihr Visual Library-Repositorium unter der Bezeichnung „Bibliotheca Mozartiana digital“ eingebracht; es handelt sich dabei um wertvolle historische Musikdrucke, Bücher und Zeitschriften, Musikhandschriften, Briefe und andere Dokumente, der im Kern auf Geschenke und Vermächtnisse von Mitgliedern der Familie Mozart zurückgeht. Bei der Erschließung dieser Bestände wird der Erfassung exemplarspezifischer Merkmale ein großes Augenmerk beigemessen. ${ }^{13}$

\subsection{Retroprojekte der OBVSG}

Die OBVSG konnte in den letzten Jahren zwei größere Retroprojekte umsetzen. 2017 wurde ein zweijähriges Retrokatalogisierungsprojekt, das 58.000 lokale Datensätze der Bibliothek der Arbeiterkammer Wien umfasst hat, abgeschlossen. ${ }^{14}$

Ein weiteres Retrodigitalisierungsprojekt, das von der Wienbibliothek im Rathaus in Auftrag gegeben worden war und das 67.800 lokale Datensätze aus dem Zeitraum 1950 bis 2011 (überwiegend Viennensia) betroffen hat, wurde 2017/2018 erfolgreich umgesetzt. ${ }^{15}$

\subsection{Weitere Aktivitäten der OBVSG}

2017 wurde von der AG Strategische Planung im Österreichischen Bibliothekenverbund eine OnlineBefragung der Leitungen der Verbundbibliotheken durchgeführt, an der sich ca. 70 Prozent der am

\footnotetext{
8 OBV (2017a).

${ }^{9}$ OBV (2018d).

${ }^{10}$ Klien (2017).

${ }^{11}$ OBV (2018d).

12 OBV (2017g).

${ }^{13}$ OBV (2017c).

14 OBV (2017d).

${ }^{15}$ OBV (2017e) und OBV (2018e).
} 
Verbund teilnehmenden Institutionen beteiligt haben. Bei den Universitätsbibliotheken betrug die Rücklaufquote sogar 90 Prozent. Abgefragt wurden sieben Sachgebiete, zu denen insgesamt rund 30 Fragen zu Dienstleistungen und Services gestellt wurden, die über die Bereitstellung eines Bibliothekssystems hinausgehen. Folgende fünf Themenfelder haben sich aus den Antworten als mögliche zukünftige Aufgabenfelder herauskristallisiert: Langzeitarchivierung als zentraler Dienst, Forschungsdatenverwaltung, Bereitstellung von Digitalisierungsdienstleistungen, der Aufbau einer Clearingstelle für bibliotheksspezifische Rechtsfragen vor allem im Zusammenhang mit Open Access an der OBVSG sowie die Entwicklung der OBVSG zu einem bibliothekswissenschaftlichen Kompetenzoder Schulungszentrum. ${ }^{16}$

Mit dem erfolgreichen Systemwechsel von Aleph 500 zu Alma hat die Verbundzentrale des Österreichischen Bibliothekenverbundes ihre Leistungsfähigkeit sehr gut unter Beweis gestellt. Ungeachtet dessen steht die OBVSG, eine Firma mit hundertprozentiger Bundesbeteiligung, vor der Herausforderung, die existenzielle Frage ihrer Finanzierung längerfristig zu lösen. Vom Bund wird nur ein seit 2002, dem Jahr der Ausgliederung, gleichbleibender Betrag in Höhe von 1,7 Millionen Euro für zentrale Leistungen (Aleph 500/Alma) bezahlt, alle seither neu entwickelten Services (z.B. Primo, Visual Library) müssen von den jeweiligen Kunden bzw. den Trägern der einzelnen Bibliotheken eigenständig finanziert werden. ${ }^{17}$

Wie in den vergangenen Jahren wurden die mittlerweile zu einer guten Tradition gewordenen und von der OBVSG organisierten Verbundtage auch in den letzten zwei Jahren erfolgreich abgehalten, wobei aus naheliegenden Gründen jeweils das neue Bibliotheksverbundsystem Alma im Fokus der Vorträge und Diskussionen stand. Am 17. Mai 2017 war die Universitäts- und Landesbibliothek Tirol Gastgeberin des 13. Verbundtages, ${ }^{18}$ am 15. Mai 2018 wurde der 14 . Verbundtag an der Universitätsbibliothek Klagenfurt durchgeführt. ${ }^{19}$

\section{Kooperation E-Medien Österreich}

Die Kooperation E-Medien Österreich (KEMÖ) ist eine freiwillig abgeschlossene Kooperation von Bibliotheken bzw. deren Trägerinstitutionen für die gemeinsame kostengünstige Lizenzierung von elektronischen Zeitschriften und Datenbanken. In der laufenden 5. KEMÖ-Vertragsperiode (Laufzeit 2018-2020) nutzen 57 Institutionen die Vorteile einer gemeinsamen Lizenzierung von elektronischen Produkten, wobei18 öffentliche Universitäten und acht Privatuniversitäten, 18 Fachhochschulen, die

\footnotetext{
${ }^{16}$ OBV (2017h) und Schiller (2018).

${ }^{17}$ Bauer und Hamedinger (2018).

18 OBV (2017b).

19 OBV (2018c) und Kronschläger (2018c).
} 
Österreichische Nationalbibliothek sowie zwölf sonstige öffentliche und private Informations-, Forschungs- und Bildungseinrichtungen mit Sitz in Österreich Mitglieder der KEMÖ sind.

In der aktuellen Vertragsperiode erfolge eine personelle Aufstockung der Geschäftsstelle der KEMÖ, die als Abteilung der OBVSG zugeordnet ist, um eine Mitarbeiterin, sodass diese nunmehr aus vier Personen besteht. Das aktuelle Portfolio der KEMÖ umfasst etwa 70 Produktverträge, die überwiegend als Mehrjahresverträge abgeschlossen wurden. Neben Marktsondierung und Verhandlungsführung sowie Abstimmung der jeweils interessierten Konsortialpartnereinrichtungen gehört auch die Abwicklung der Vergabeverfahren zu den zentralen Aufgaben der Geschäftsstelle der KEMÖ. Seit der aktuellen Vertragsperiode liegt ein weiterer Tätigkeitsschwerpunkt auch auf dem Thema Open Access, was nicht zuletzt im aktuellen Hochschulraumstrukturmittelprojekt „Austrian Transition to Open Access (AT2OA)“ begründet ist. Die besondere Herausforderung hierbei sind neben optimalen Vertragsabschlüssen die Entwicklung und die laufende Optimierung von Open Access-Workflows sowie ein begleitendes laufendes Monitoring (siehe Abschnitt 6). Derzeit umfasst das Open Access-Portfolio der KEMÖ sieben Vereinbarungen, darunter jeweils auf drei Jahre abgeschlossene kombinierte Open Access-Publikations- und Subskriptionsverträge mit den Verlagen Springer Nature bzw. Wiley. ${ }^{20}$

\section{3 Österreichische Nationalbibliothek}

\subsection{Vision 2025}

Im Herbst 2012 wurde von der Österreichischen Nationalbibliothek ihre „Vision 2025 - Wissen für die Welt von morgen“ vorgestellt, die von den grundlegenden gesellschaftlichen Werten - freier Zugang zum Wissen, Innovation, Bildung, Verantwortung - getragen wird. Darauf aufbauend wurden als wesentliche Themenfelder des Strategieentwicklungsprozesses für die Jahre 2017 bis 2021 die Modernisierung der IT-Infrastruktur und die Ablöse des lokalen Bibliothekssystems Aleph 500 durch ALMA sowie als weitere Schwerpunkte Digitalisierung und Portale, Linked Open Data, Digital Humanities und Crowdsourcing festgelegt. ${ }^{21}$ In diesem Zusammenhang ist die erste CrowdfundingAktion zu nennen, die für die Restaurierung eines Prachtbandes zu Maria Theresia durchgeführt wurde und die sehr gut verlaufen ist. ${ }^{22}$

Eine wichtige Rolle kommt auch den Online-Aktivitäten der Nationalbibliothek zu - vom virtuellen Prunksaal über den neuen Webauftritt bis zum Forschungsblog. ${ }^{23}$

\footnotetext{
${ }^{20}$ OBV (2018b).

${ }^{21}$ Mayr (2018).

22 Zauner (2017).

${ }^{23}$ Schmidt (2018).
} 


\subsection{Erforschung von Teilbeständen und Sammlungen der Österreichischen Nationalbibliothek}

In einer Reihe von Projekten werden von der Österreichischen Nationalbibliothek laufend Teilbestände und einzelne Sammlungen erforscht und inhaltlich erschlossen. Die Themenpalette reicht von der Habsburg-Lothringischen Familien-Fideikommissbibliothek ${ }^{24}$ über die Sammlungen Emile Zuckerkandl, ${ }^{25}$ das Digitalisierungsprojekt AKON - Ansichtskarten Online ${ }^{26}$ und die frauen- und geschlechterspezifischen Bestände ${ }^{27}$ bis zu Projekten des Literaturmuseums ${ }^{28}$ und des Esperantomuseums. ${ }^{29}$

\subsection{Weitere Aktivitäten der Österreichischen Nationalbibliothek}

Die Österreichische Nationalbibliothek hat in den 2017 und 2018 bedeutende Materialien als Geschenk oder durch Kauf in ihren Bestand übernommen, darunter die literarischen Nachlässe von Trude Marzik (1923-2016) ${ }^{30}$ und Frederic Morton (1924-2015), ${ }^{31}$ einen Teilnachlass von Ingeborg Bachmann (1926-1973) ${ }^{32}$ sowie den Vorlass der 1931 geborenen Ruth Klüger. ${ }^{33}$

Anlässlich des 650-Jahr-Jubiläums der Österreichischen Nationalbibliothek hat der Österreichische Rundfunk (ORF), vertreten durch seinen Generalsekretär Alexander Wrabetz, am 14. November 2017 die Patenschaft für drei ausgewählte Werke mit Bezug zur Geschichte des ORF übernommen. ${ }^{34}$ Im Zuge der Sanierungsarbeiten am Parlamentsgebäude wird das Parlament vorübergehend auch in Räumlichkeiten der Hofburg untergebracht, weshalb im Vorfeld die Ausbildungsabteilung der Österreichischen Nationalbibliothek voraussichtlich für die Dauer von drei Jahren aus der Hofburg ausgesiedelt wurde. Provisorischer Standort seit 1. Februar 2017 ist das Gebäude der ehemaligen Wirtschaftsuniversität Wien. ${ }^{35}$

Mit Jahresende 2017 hat die Österreichische Nationalbibliothek ihr Fortbildungsprogramm „Brain Pool" wegen zu geringer Nachfrage und mangelnder Kostendeckung eingestellt.

Im Prunksaal der Österreichischen Nationalbibliothek wurden 2017 zwei Ausstellungen eröffnet. Von Februar bis Juni 2017 wurde anlässlich des 300. Geburtstages die Ausstellung „Maria Theresia:

\footnotetext{
${ }^{24}$ Ortner (2017) sowie Knieling et al. (2018).

${ }^{25}$ Brantl (2018).

${ }^{26}$ Müller et al. (2017).

27 Bittermann-Wille und Jammernegg (2017).

28 Manojlovic (2017).

29 Tuider (2017).

30 Pauser (2017c).

${ }^{31}$ Nationalbibliothek hat Morton-Nachlass und Klüger-Vorlass bekommen (2018).

${ }^{32}$ Nationalbibliothek ersteigert Teilnachlass Ingeborg Bachmanns (2018) und Jandl (2018).

${ }^{33}$ Nationalbibliothek hat Morton-Nachlass und Klüger-Vorlass bekommen (2018).

34 ORF übernimmt Patenschaften über drei Bücher zur Geschichte des ORF (2017).

35 Pauser (2017b).
} 
Habsburgs mächtigste Frau" gezeigt. ${ }^{36}$ Von Juni 2017 bis Januar 2018 folgte eine Ausstellung über „300 Jahre Freimaurer: Das wahre Geheimnis“, die vom österreichischen Bundespräsidenten a. D. Heinz Fischer am 22. Juni 2017 eröffnet wurde. ${ }^{37}$

\subsection{0-jähriges Jubiläum der Österreichischen Nationalbibliothek}

Im Jahr 2018 wurde von der Österreichischen Nationalbibliothek unter Bezugnahme auf das 1368 angefertigte „Evangeliar des Johannes von Troppau“ das 650-jährige Jubiläum in zahlreichen Veranstaltungen gefeiert. Diese in Goldlettern geschriebene und reich illustrierte prachtvolle Handschrift, die sich im Besitz des Habsburgerherzogs Albrecht III. befunden hat, gilt als Gründungscodex der Nationalbibliothek.

Der Prunksaal war das ganze Jahr über geprägt von der großen Jubiläumsausstellung „Schatzkammer des Wissens, 650 Jahre Österreichische Nationalbibliothek“, die am 25. Januar 2018 von Gernot Blümel, Bundesminister für EU, Kunst, Kultur und Medien, eröffnet wurde. ${ }^{38} \mathrm{Im}$ Rahmen der Ausstellung, die von 26. Januar 2018 bis 13. Januar 2019 dauerte, wurden die Schätze der Bibliothek, Prachthandschriften und wertvolle Frühdrucke, kostbare Musiknoten, Landkarten ebenso wie Fotos und Grafiken präsentiert. ${ }^{39}$ Besonders attraktive Stücke aus der Nationalbibliothek wurden im Jubiläumsjahr als „Objekt des Monats“ jeweils nur für einen kurzen Zeitraum gezeigt. Darunter befanden sich neben dem „Evangeliar des Johannes von Troppau“ auch eine Papyrusurkunde zum Indienhandel, die „Gutenberg-Bibel“, Mozarts „Requiem“ und das handschriftlich korrigierte Typoskript von Ingeborg Bachmanns Gedicht „Böhmen liegt am Meer".

Eingeleitet wurden die zahlreichen Aktivitäten der Österreichischen Nationalbibliothek anlässlich des 650-Jahr-Jubiläums - von der Jubiläumsausstellung über eine Sonderausstellung zu „Berg,

Wittgenstein, Zuckerkandl“ im Literaturmuseum und ein Open House bis zu einem Symposium - von einem feierlichen Festakt, der am 22. Februar 2018 stattfand. ${ }^{40}$ Aus diesem Anlass sprach Generaldirektorin Johanna Rachinger über die Geschichte der Nationalbibliothek, über deren Aktivitäten im Jubiläumsjahr und über deren Perspektiven angesichts der Herausforderungen der digitalen Revolution. ${ }^{41}$ Die Festveranstaltung wurde auch durch die Anwesenheit des österreichischen Bundespräsidenten Alexander von der Bellen beehrt, der in einer Rede seine hohe Wertschätzung für die Österreichische Nationalbibliothek zum Ausdruck brachte. ${ }^{42}$

\footnotetext{
${ }^{36}$ Schau zu 300 Jahre Maria Theresia in der Nationalbibliothek (2017).

37 Baumgartner (2017).

${ }^{38}$ Bundeskanzleramt/Bundesregierung/Nachrichten (2018).

39 Österreichische Nationalbibliothek Prunksaal: Schatzkammer des Wissens und Wurmitzer (2018).

${ }^{40}$ Festakt zum 650-Jahr-Jubiläum der Nationalbibliothek (2018).

${ }^{41}$ Rachinger (2018).

42 Van der Bellen (2018).
} 


\section{$4 \quad$ Forum Universitätsbibliotheken Österreichs}

2017 und 2018 wurden im Rahmen des Forums Universitätsbibliotheken Österreichs (ubifo), einem Kooperationsgremium der Leiterinnen und Leiter der 20 Universitätsbibliotheken der öffentlichen Universitäten sowie der Österreichischen Nationalbibliothek, je drei Meetings durchgeführt (15.03.2017 an der Wirtschaftsuniversität Wien, 16.05.2017 an der Universitäts- und Landesbibliothek Tirol, 09.11.2017 an der Veterinärmedizinischen Universität Wien, 08.03.2018 an der Technischen Universität Wien, 07.06.2018 an der Universität Wien und am 15.11.2018 an der Universitätsbibliothek Salzburg).

Im Fokus des Forums stand in den vergangenen zwei Jahren vor allem der Umstieg auf das neue Bibliothekssystem ALMA und dessen Auswirkungen auf die Abläufe an den einzelnen Bibliotheken, aber auch auf den Verbund in seiner Gesamtheit. In diesem Zusammenhang wurde auch das Thema Datenschutzgrundverordnung (DSGVO) wiederholt auf die Agenda gesetzt. Besprochen wurden auch die Ergebnisse der beiden Umfragen „Zukünftige Dienstleistungen der OBVSG“ (siehe Abschnitt 1) sowie „Prekäre Arbeitsverhältnisse im österreichischen Bibliothekswesen“ aus der Sicht der Universitätsbibliotheken.

Weitere wichtige Themen waren Berichte zu den Hochschulraumstukturmittelprojekten „EInfrastructures Austria“ bzw. „E-Infrastructures Austria plus“ (siehe Abschnitt 5), „Austrian Transition to Open Access (AT2OA)“ (siehe Abschnitt 6) und "Open Education Austria (OEA)“ sowie zu den neuen Initiativen „Austrian Open Science Support Group“ , „European Open Science Cloud" und „GO FAIR".

Ein Thema, das regelmäßig auf der Agenda des Forums Universitätsbibliotheken Österreichs stand, war Status und Fortschritte bei der Erstellung des KEMÖ Folgevertrages 2018-2020, bei dem neben der Aufstockung der Geschäftsstelle und einem damit einhergehenden neuen Kostenschlüssel vor allem die Zusammensetzung des Kooperationsausschusses besprochen wurde (siehe Abschnitt 2).

Erörtert wurden auch die aktuellen Entwicklungen zum Urheberrecht sowie die Zukunft der bibliothekarischen Fortbildung nach der Einstellung des „Brain Pool“-Programmes der Österreichischen Nationalbibliothek mit Jahresende 2017.

Neben der vom Forum Universitätsbibliotheken Österreichs bereits 2010 gestarteten Initiative „Gemeinsame Archivierung an österreichischen Universitätsbibliotheken“, die das Ziel verfolgt, die Aufbewahrung von zumindest einem Printexemplare österreichweit von elektronisch erworbenen Zeitschriften sicherzustellen und durch das System der verteilten Archivierung eine gleichmäßige Aufteilung der Belastung zu erreichen, ${ }^{43}$ wurde 2017 mit der Etablierung des „Netzwerk

\footnotetext{
${ }^{43}$ Kromp und Mayer (2017).
} 
RepositorienmanagerInnen (RepManNet)" ein weiterer wichtiger Akzent gesetzt. Das Netzwerk versteht sich als Kommunikationsplattform für Institutionen in ganz Österreich, die ein Repositorium betreuen und sich mit anderen über Erfahrungen, Herausforderungen und Spezialthemen austauschen möchten (siehe Abschnitt 5). ${ }^{44}$

\section{$5 \quad$ Forschungsdaten und E-Infrastructures Austria}

Nach dem Ende der Laufzeit von „E-Infrastructures Austria“, einem vom Bundesministerium für Wissenschaft, Forschung und Wirtschaft (BMWFW) finanzierten Hochschulraumstrukturmittelprojekt (Laufzeit 2014-2016), ${ }^{45}$ wurde vom BMWFW auch das Folgeprojekt „E-Infrastructures Austria Plus" bewilligt, in dessen Rahmen Maßnahmen im Kontext von Forschungsdatenmanagement an neun Universitäten gefördert werden. Das Projekt, dessen Leitung an der Universität Innsbruck liegt und dem sich neben den antragsstellenden Einrichtungen bereits sechs weitere Institutionen als assoziierte Projektpartner angeschlossen haben, hat eine Laufzeit von 2017 bis 2019 . „EInfrastructures Austria Plus" verfolgt das Ziel, eine Infrastruktur für e-Science in Österreich zu schaffen, wobei es sich auf ein österreichisches Netzwerk stützt und dieses weiterentwickelt, in dem Ressourcen und vorhandenes Expertenwissen gebündelt wird. Durch die Kooperation im Projekt sollen Effizienzsteigerungen im Bereich Sicherung, Archivierung und Zugänglichmachung von digitalen Ressourcen und Forschungsdaten erzielt, der Nutzen von Forschungsdaten für Studierende und Lehrende verbessert, die Teilnahme an universitätsübergreifenden IT-basierten Verwaltungsnetzten gefördert und der gemeinsame Nutzen von Wissen über Prozesse und Technologien in einem immaterialgüterrechtlich geordneten Rahmen ermöglicht werden. „E-Infrastructures Austria Plus" gliedert sich in die sieben Arbeitspakete Research Lifecycle, Forschungsdatenmanagement Policy, Datenmanagement Pläne, institutionelle Repositorien, Metadaten, persistente Identifikatoren und Lab Notebook. Ergänzt werden die Arbeitspakete um eine Initiative, die innerhalb des Projektes das Ziel verfolgt, GO FAIR (Findable, Accessible, Interoperable und Reusable) und dessen Ziel, eine faire Nutzung von Forschungsdaten zu realisieren, an Forschungsinstitutionen in Österreich auf lokaler Ebene zu verankern und den Aufbau von Reference Points sowie von Infrastrukturen und forschungsunterstützenden Services zu unterstützen.

\footnotetext{
${ }^{44}$ Torggler und Andrae (2018).

45 Bauer et al. (2017).
} 


\section{Open Access und AT2OA}

\subsection{Open Access Network Austria}

Das Open Access Network Austria (OANA), dem Wissenschaftlerinnen und Wissenschaftler sowie Vertreterinnen und Vertretern von Forschungsförderinstitutionen, Rektoraten und Universitätsbibliotheken sowie des für Wissenschaft und Forschung zuständigen Bundesministeriums angehören, kommt seit seiner Gründung im Jahr 2012 eine wichtige Rolle für die Verankerung von Open Access Initiativen und Projekten in Österreich zu. ${ }^{46}$ Am 10. Januar 2018 wurde die 4. OANAInformationsveranstaltung im Palais Harrach in Wien veranstaltet, an dem zirka 70 Personen aus den beteiligten Einrichtungen teilgenommen haben. Einem kurzen Rückblick auf die Ereignisse und aktuellen Entwicklungen von Open Access in Österreich folgte die Präsentation der Ergebnisse der einzelnen OANA Arbeitsgruppen (AG Open Access \& Open Educational Resources, AG Strategische Positionierung \& Administration der Open-Access-Transition, AG Open Access \& die Zukunft von Scholarly Communication). Im Rahmen der Veranstaltung wurde auch bekräftigt, dass sich OANA als ein Thinktank zum Thema Open Science, mit einer offenen Struktur und ohne Rechtsform versteht. Ziele sind der Austausch von Ideen, Koordination und Vernetzung von Initiativen sowie die Erarbeitung von Empfehlungen zu Open Science. ${ }^{47}$

\subsection{Austrian Transition to Open Access(AT2OA)}

Im Rahmen der Förderung von Kooperationsprojekten an Hochschulen wurde vom BMWFW auch ein Projekt mit der Zielsetzung bewilligt, Open Access in Österreich zu fördern. Das Hochschulraumstrukturmittelprojekt „Austrian Transition to Open Access (AT2OA)“ (Laufzeit 20172020), an dem alle 21 öffentlichen Universitäten Österreichs kooperieren, verfolgt das Ziel, Open Access durch geeignete, gemeinsam abgestimmte Maßnahmen voranzubringen, und gliedert sich in vier Teilprojekte. Im Teilprojekt 1 „Analyse der Auswirkung einer Umstellung auf Open Access“ werden die finanziellen Auswirkungen einer vollständigen Transformation zu Open Access für die am Projekt beteiligten Einrichtungen analysiert. In Teilprojekt 2 „Finanzierung von Open AccessÜbergangsmodellen“ steht die Erweiterung von subskriptionsbasierten Lizenzverträgen um Open Access-Komponente auf der Agenda. Der „Auf-, Ausbau und Finanzierung von Open AccessPublikationsfonds“ ist Ziel von Teilprojekt 3. Teilprojekt 4 dient der „Förderung von OA-Publikationen und alternativen OA-Publikationsmodellen von Universitäten“. ${ }^{48}$

\subsection{Open Access Vereinbarungen mit Modellcharakter}

Im Rahmen des Teilprojekts 2 von AT2OA wurde über die Kooperation E-Medien Österreich (KEMÖ) analog zu dem bereits 2016 abgeschlossenen Springer Compact-Lizenzvertrag eine modellhafte

\footnotetext{
${ }^{46}$ Bauer (2017).

${ }^{47}$ Reckling und Rieck (2018).

${ }^{48}$ Bauer (2017a) sowie Bauer et al. (2018).
} 
Vereinbarung mit dem Verlag Wiley abgeschlossen. Während der Vertragslaufzeit von 2018 bis 2020 haben alle Forschenden und Studierenden der beteiligten 22 Einrichtungen die Möglichkeit, die Inhalte aller von Wiley herausgegebenen Subskriptions-Zeitschriften zu lesen und in diesen ihre Forschungen kostenfrei Open Access zu veröffentlichen, sofern sie für die betreffenden Artikeln als Corresponding Author fungieren. ${ }^{49}$

In Österreich gab es 2018 mit neun Verlagen Open Access-Abkommen zur Veröffentlichung in Zeitschriften, wobei der Großteil dieser Verträge von der Kooperation E-Medien Österreich koordiniert wird (BioMedCentral, Emerald, Frontiers, IOP Publishing, Royal Society of Chemistry, SAGE, Springer Nature, Taylor and Francis, Wiley).

Ein wesentlicher Aspekt der Transition-Verträge liegt in der laufenden Überprüfung, in welchem Maß die damit verbundenen Ziele auch tatsächlich erreicht werden; eine erste Zwischenbilanz für den Springer Compact-Vertrag wurde 2017 gezogen. ${ }^{50} 2018$ wurde auch eine erste Analyse aller bestehenden Lizenzverträge mit Open Access-Komponente vorgelegt, in der überprüft wurde, welche Auswirkungen die unterschiedlichen Verträge auf die jeweiligen Publikationszahlen haben. ${ }^{51}$

\subsection{Repositorien}

In den letzten Jahren hat sich die Zahl der an österreichischen Forschungseinrichtungen betriebenen Repositorien im Zusammenhang mit dem Hochschulraumstrukturmittelprojekt „E-Infrastructures Austria" deutlich erhöht. 2013, im Jahr vor Beginn des Projektes, waren 13 österreichische Repositorien im DOAJ verzeichnet, diese Zahl erhöhte sich bis Ende 2016 auf 28; Ende 2018 sind 30 österreichische Repositorien im DOAJ registriert. Eine ähnlich positive Entwicklung zeigt sich in re3data.org (Registry of Research Data Repositories), in dem 32 Repositorien für Forschungsdaten, die in Österreich bzw. in Kooperation mit in Österreich ansässigen Einrichtungen betrieben werden, erfasst sind. ${ }^{52}$

Bereits 2015 wurde im Rahmen einer Telefonumfrage zum Thema Metadaten eine Bestandsaufnahme der an den einzelnen Universitäten im Einsatz befindlichen Repositorien gemacht, um einen Überblick über die wichtigsten Softwaresysteme und deren spezifische Einsatzzwecke, die am häufigsten verwendeten Metadatenstandards und die Gepflogenheiten bzw. Erwartungen der Nutzerinnen und Nutzer zu gewinnen. ${ }^{53}$

\footnotetext{
${ }^{49}$ Wiley (2018).

${ }^{50}$ Andrae undVillányi (2017).

51 Villányi (2018).

52 Bauer und Ferus (2018).

${ }^{53}$ Blumesberger und Zartl (2017).
} 
Die Palette der Repositorien reicht von Phaidra, dem Repositorium der Universität Wien, ${ }^{54}$ über unipub $^{55}$ und GAMS ${ }^{56}$ an der Universität Graz, ARCHE an der Österreichischen Akademie der Wissenschaften, ${ }^{57}$ IST PubRep und IST DataRep am IST Austria, ${ }^{58}$ VOLARE an der Vorarlberger Landesbibliothek ${ }^{59}$ bis zu Visual Library-Anwendungen im Österreichischen Bibliothekenverbund, ${ }^{60}$ beispielweise dem Repository der Universität Mozarteum Salzburg. ${ }^{61}$

Anlässlich des 10-jährigen Jubiläums von Phaidra, dem Repositorium der Universität Wien, fand am 24. Oktober 2017 eine Tagung statt, in deren Rahmen das Thema Repositorium aus der Sicht der Wissenschaft, aber auch aus der Sicht der betreibenden Bibliothek dargestellt und diskutiert wurde. ${ }^{62}$

\section{$7 \quad$ NS-Provenienzforschung}

2017 und 2018 fanden Restitutionen aus Beständen der Universitätsbibliothek Salzburg, der Universitätsbibliothek der Wirtschaftsuniversität Wien und der Österreichischen Nationalbibliothek statt.

Am 31. Januar 2017 restituierte die Universitätsbibliothek Salzburg, die in einem dreijährigen Projekt, finanziert von Universität und Land Salzburg, zwischen 2009 und 2012 insgesamt 240.000 Bänden aus ihrem Bestand einer Autopsie unterzogen hatte, zehn handschriftliche Manuskripte und 40 gedruckte Bücher aus dem Konradinum, einem Pflegeheim für Menschen mit schwerer geistige oder mehrfacher Behinderung, in Eugendorf an das Land Salzburg. Die Bücher waren nach der 1938 erfolgten Beschlagnahme des Konradinums durch die Nationalsozialisten als unrechtmäßige Erwerbungen an die Universitätsbibliothek Salzburg gelangt. ${ }^{63}$

Bereits am 1.Oktober 2015 restituierte die Wirtschaftsuniversität Wien die aus 696 Bänden bestehende Privatbibliothek des österreichischen Petrochemikers Leopold Singer (1869-1942) an dessen Erbinnen und Erben, die sie an das Technische Museum Wien übergaben, wo sie in einer Dauerausstellung gezeigt werden. Im Rahmen eines Festaktes, der anlässlich der Übergabe und der nunmehrigen Präsentation am 4. Oktober 2017 am Technischen Museum stattgefunden hat, erfolgte die Restitution eines weiteren Buches, das sich bis zur nationalsozialistischen Machtergreifung in Österreich im Besitz von Martin Singer (1874-1959), dem jüngeren Bruder von Leopold, befunden

\footnotetext{
${ }^{54}$ Blumesberger (2018), Eberhard und Kraus (2018), Hagmann (2018) sowie Heider (2018).

55 Kaier und Schilhan (2018).

${ }^{56}$ Stigler und Steiner (2018).

57 Trognitz und Ďurčo (2018).

58 Petritsch und Porsche (2018).

${ }^{59}$ Eberle und Feurstein (2018).

60 Wiese (2018).

${ }^{61}$ Buland und Leitner (2018).

62 Blumesberger (2017).

63 Lahner (2017).
} 
hatte, durch die Wirtschaftsuniversität Wien, wo seit 2010 NS-Provenienzforschung betrieben wird. Nach 1938 konnten die Brüder Singer nach Großbritannien bzw. Australien emigrieren, ihre Bücher allerdings nicht mitnehmen. Diese wurden von der Gestapo beschlagnahmt und gelangten wahrscheinlich 1942 als unrechtmäßiger Erwerb an die Hochschule für Welthandel, der Vorgängerin der heutigen Wirtschaftsuniversität. Mit der Übergabe der Bestände an das Technische Museum Wien wurde erstmals im deutschsprachigen Raum ermöglicht, Ergebnisse bibliothekarischer NSProvenienzforschung in einem Museum der Öffentlichkeit zugänglich zu machen. ${ }^{64}$

2.255 in der NS-Zeit unrechtmäßig erworbene „erblose“ Bücher wurden von der Österreichischen Nationalbibliothek gemäß Kunstrückgabegesetz an den Nationalfonds der Republik Österreich für Opfer des Nationalsozialismus restituiert und anschließend für rund 75.000 Euro zurückgekauft. Bei der Gedenkfeier, die am 16. Dezember 2018 im Oratorium der Nationalbibliothek stattgefunden hat, war auch der Vorsitzende des Kuratoriums des Nationalfonds, Nationalratspräsident Wolfgang Sobotka, anwesend. ${ }^{65}$

Von 2. bis 4. Mai 2017 fand an der Universität Wien eine Tagung zum Thema „Treuhänderische Übernahme und Verwahrung“ statt, die von der „Arbeitsgruppe NS-Provenienzforschung“ der Vereinigung Österreichischer Bibliothekarinnen \& Bibliothekare und vom „Arbeitskreis Provenienzforschung und Restitution - Bibliotheken“ veranstaltet wurde. ${ }^{66}$

Unter dem Titel "NS-Provenienzforschung und Restitution an Bibliotheken" wurde 2017 von drei österreichischen Bibliothekaren ein praxisbezogener Leitfaden mit der Zielsetzung herausgegeben, Bibliotheksmitarbeiterinnen und -mitarbeiter sowie sonstige interessierte Personen in die Problematik der NS-Provenienzforschung einzuführen und mit wesentlichen Informationen auszustatten, um bedenkliche Fälle in der alltäglichen Arbeit zu erkennen und zu behandeln. ${ }^{67}$

\section{Einzelaktivitäten von Bibliotheken}

\subsection{Umbau der Universitätsbibliothek Graz}

Als erste Maßnahme im Zuge der Sanierung und der Erweiterung der Universitätsbibliothek Graz erfolgte im Sommer 2016 die Übersiedlung der Abteilungen der Bibliothek und der Bestände aus dem alten Bibliotheksgebäude der Universität Graz in entsprechend adaptierte Ersatzquartiere. Danach wurden die großen Anlagen und Installationen, wie Regalanlagen, Aggregate der Klimaanlagen und Lifte, demontiert und entsorgt. Zwischen April 2017 und Juni 2018 erfolgten der Abriss des Verwaltungstrakts der Universitätsbibliothek, eines Zubaus von 1970, der Bau eines

\footnotetext{
${ }^{64}$ Koll und Zodl (2017).

65 ÖNB restituierte 2255 Bücher an den Nationalfonds (2018).

${ }^{66}$ Alker-Windbichler und Bauer (2017) sowie Kaiser et al. (2017).

${ }^{67}$ Alker-Windbichler et al. (2017).
} 
unterirdischen Hörsaals mit einer Kapazität von 430 Sitzplätzen und der Aufbau eines zweigeschossigen Glasquaders mit 200 Studierendenarbeitsplätzen über dem historischen Lesesaal. Im Juni 2018 konnte die Gleichenfeier begangen werden. Die Eröffnung der umgestalteten Bibliothek soll im Herbst 2910 am alten Standort erfolgen. ${ }^{68}$

\subsection{Publikationsunterstützung der Universitätsbibliothek Graz}

An der Universitätsbibliothek Graz wurden eine Stabsstelle Publikationsservices eingerichtet, um den Wissenschaftlerinnen und Wissenschaftlern der Universität Services zur Unterstützung des wissenschaftlichen Publizierens und der Sichtbarmachung wissenschaftlicher Forschungsleistung anzubieten. Im Rahmen dieser neuen Aufgabe wird von der Universitätsbibliothek Graz in den Bereichen Universitätsverlag, Open-Access-Repositorium, Bibliometrie, Open-Access-Abkommen und Forschungsdatenmanagement Hilfestellung geleistet. Die neue Stabsstelle, deren Services im April 2016 universitätsweit angekündigt worden sind, kooperiert auch mit anderen Einrichtungen der Universität, die in den Bereichen Forschungsunterstützung und -bewertung tätig sind, darunter Forschungsmanagement und -service sowie Leistungs- und Qualitätsmanagement. ${ }^{69}$

\subsection{Zertifizierung gemäß ISO 9001 an der Universitätsbibliothek der Medizinischen Universität Wien}

Der Zielsetzung verpflichtet, ihre Produkte und Services laufend zu optimieren, unterzieht sich die Universitätsbibliothek der Medizinischen Universität Wien seit 2012 einem kontinuierlichen Zertifizierungsverfahren gemäß der internationalen Norm DIN EN ISO 9001. Auch wenn Planung, Aufbau und Etablierung sowie Weiterentwicklung eines Qualitätsmanagementsystems gemäß dieser Norm bedeutende Ressourcen, vor allem personeller Natur, beanspruchen, stehen diesem Einsatz sehr positive Effekte auf die erstellten Produkte und angebotenen Services gegenüber. Zu nennen sind hier eine bessere Erfüllung von Kundenwünschen, eine höhere Transparenz bei Arbeitsabläufen und Prozessen sowie eine effizientere Ermittlung und Beseitigung von Fehlerquellen. Sieben Jahre nach der Einführung eines Qualitätsmanagementsystems gemäß ISO 9001 hat sich an der Universitätsbibliothek der Medizinischen Universität Wien bestätigt, dass dieses Instrument zur Implementierung und Weiterentwicklung eines ständigen Verbesserungsprozesses, auch an einer wissenschaftlichen Bibliothek, sehr gut geeignet ist. ${ }^{70}$

\subsection{Bibliotheksporträt österreichischer Landesbibliotheken}

In „Bibliothek Forschung und Praxis“ wurden 2017 und 2018 drei österreichische Landesbibliotheken vorgestellt, wobei in den Darstellungen die jeweilige historische Entwicklung im Vordergrund stand.

\footnotetext{
68 Schlacher (2018).

69 Lackner und Ginther (2018) sowie Kaier und Lackner (2018).

70 Bauer (2017d) und Bauer (2018d).
} 
Die Steiermärkische Landesbibliothek, 1811 von Erzherzog Johann als Leseanstalt gegründet, ist mit einem Bestand von 750.000 Bänden die größte und älteste Landesbibliothek Österreichs. Nach einem Umbau wurde die völlig neu gestaltete Landesbibliothek 2012 am alten Standort im Joanneumsviertel wiedereröffnet. ${ }^{71}$

Die 1813 in Wien, der früheren Hauptstadt von Niederösterreich, gegründete Niederösterreichische Landesbibliothek wurde 1997 in einen Neubau in die neue Landeshauptstadt Sankt Pölten übersiedelt. Die Bibliothek verfügt heute bei einem jährlichen Zuwachs von ca. 15000 Bänden über einen Bestand von 380000 Bänden. ${ }^{72}$

Die 1921 gegründete Burgenländische Landesbibliothek präsentiert sich heute als Forschungsbibliothek, die über einen Bestand von zirka 140.000 Bänden verfügt und sich vor allem durch ihren historischen Schwerpunkt auszeichnet, zu dem auch Schriftgut aus dem vormaligen Königreich Ungarn zählt. ${ }^{73}$

\section{BID-Ausbildung in Österreich}

Den 13. Grundlehrgang des interuniversitären Universitätslehrgangs Library and Information Studies, der im Studienjahr 2016/2017 an der Universität Wien in Kooperation mit der Österreichischen Nationalbibliothek durchgeführt wurde, konnten 22 Studierende (16 Studentinnen und 6 Studenten) erfolgreich abschließen.

Im Studienjahr 2017/2018 absolvierten 24 Studierende an der Universitätsbibliothek Wien und 22 Studierende an der Österreichischen Nationalbibliothek den 14. Grundlehrgang des Interuniversitären Universitätslehrgangs Library and Information Studies.

Im Rahmen der Ausbildungen 2016/2017 bzw. 2017/2018 wurden insgesamt 27 Abschlussprojekte umgesetzt, in deren Rahmen u.a. Bestände des Literaturarchivs der Österreichischen Nationalbibliothek, der Fachbibliothek des Bibliotheksverbandes Österreichs (BVÖ), der Landesbibliothek Oberösterreich, der Bibliothek des Salzburg Museums, einer Forschungsbibliothek des Stiftes Heiligenkreuz, der Bibliothek des Österreichischen Patentamtes und des ORF-Archivs bearbeitet wurden.

Im Wintersemester 2018/2019 begann der 15. Grundlehrgang des Interuniversitären Universitätslehrgangs Library and Information Studies mit 26 Studierenden an der Universitätsbibliothek Wien und 22 Studierenden an der Österreichischen Nationalbibliothek.

\footnotetext{
${ }^{71}$ Kocher-Lichem (2017).

72 Alscher (2017).

73 Perschy (2018).
} 
2017 und 2018 beendeten zehn Teilnehmerinnen des ersten Masterlehrgangs Library and Information Studies nach Curriculum 2013, der von der Universität Wien in Kooperation mit der Österreichischen Nationalbibliothek durchgeführt wurde, ihre Ausbildung.

Der im Studienjahr 2017/2018 in Kooperation des Ausbildungslehrgangs Bibliothek, Information und Dokumentation mit den Universitäten Wien und Innsbruck angebotene Lehrgang für die Tätigkeitsbereiche mittlerer Qualifikation wurde von 13 Personen besucht, wobei die sechs Module abwechselnd in Wien und Innsbruck stattfanden.

2017 und 2018 beendeten insgesamt 50 Lehrlinge ihre Ausbildung im Lehrberuf Archiv-, Bibliotheksund Informationsassistent/in durch die erfolgreiche Ablegung ihrer Lehrabschlussprüfungen im Gewerbehaus der Wirtschaftskammer in Wien. ${ }^{74}$

2017 wurde eine Studie zu Arbeitsmarktperspektiven, Beschäftigungsverhältnissen und Berufszufriedenheit von Absolventinnen und Absolventen des Grundlehrgangs Library and Information Studies in Österreich veröffentlicht. Methodisch stützte sich diese Untersuchung auf eine Online-Umfrage, die sich an alle Teilnehmerinnen und Teilnehmer der Grundlehrgänge 2010 bis 2015 an allen Ausbildungsstandorten in Österreich gewendet hat, und eine Auswertung von 479 Stellenausschreibungen, die zwischen 2011 und 2015 im VÖBBLOG veröffentlicht worden sind. Die Studie bietet einen guten Einblick in die Einstiegssituation im BID-Bereich nach Abschluss der Ausbildung. ${ }^{75}$

\section{BID-Tagungen in Österreich}

\subsection{3. Österreichischer Bibliothekartag in Linz 2017}

Der 33. Österreichische Bibliothekartag, der von 12. bis 15. September 2017 an der Universität Linz stattgefunden hat, war dem Motto „Wolkenkuckucksheim. Bibliotheken in der Cloud" gewidmet. Themenschwerpunkt dieser traditionsreichen und größten bibliotheksspezifischen Fortbildungsveranstaltung in Österreich, die von der Vereinigung Österreichischer Bibliothekarinnen und Bibliothekare (VÖB), dem Büchereiverband Österreichs (BVÖ) und der Universitätsbibliothek Linz veranstaltet wurde, waren die künftige Rolle der Bibliotheken in Forschung und Lehre (I), Personalund Organisationsentwicklung als Notwendigkeit in zukunftsorientierten Bibliotheken (II), der Einfluss der technischen Entwicklung auf die Informationsversorgung (III), Bibliotheken im Spannungsfeld politischer und wirtschaftlicher Rahmenbedingungen (IV), traditionelle Aufgaben neue Dienstleistungen (V) und die Bibliothek als Dritter Ort (VI). ${ }^{76}$

\footnotetext{
${ }^{74}$ Pum (2018).

75 Nowak und Schneckenleithner (2017).

${ }^{76}$ Franke et al. (2018).
} 


\subsection{Informationskompetenz-Tagung in Innsbruck 2017}

Am 16. und 17. Februar 2017 fand an der Universitäts- und Landesbibliothek Tirol die „1. Informationskompetenz-Tagung Deutschland/Österreich“ und zugleich die erste überregionale Tagung im deutschsprachigen Raum zum Thema Informationskompetenz statt, die von 110 Bibliothekarinnen und Bibliothekare aus Österreich und Deutschland besucht wurde. ${ }^{77}$

\subsection{Digitale Bibliothek in Graz 2017}

Bereits zum 7. Mal fand am 2. und 3. März 2017 die Tagung „Digitale Bibliothek. Zukunft des digitalen Erbes? Nachhaltige Lösungen für Gedächtnis- und Wissenschaftseinrichtungen" statt, die sich den Fragen der nachhaltigen Gestaltung von Datenarchivierung, Infrastrukturen, Services und Partnerschaftsmodellen für die zukünftigen Anforderungen in einer digitalisierten Lebenswelt gestellt hat. $^{78}$

\subsection{AGMB Jahrestagung in Wien 2017}

Von 25. bis 27. September 2017 war die Veterinärmedizinische Universität Wien Gastgeberin für die traditionsreiche Jahrestagung der Arbeitsgemeinschaft für Medizinisches Bibliothekswesen (AGMB), der größten fachspezifischen Bibliothekarsvereinigung im deutschsprachigen Raum.

Themenschwerpunkte der Tagung, die dem Motto „Medizinbibliotheken: inter:disziplinär inter:national - inter:aktiv" gewidmet war, waren Informationsdienste, Informationskompetenz und Nutzerschulung, Informationsvermittlung und Open Access. ${ }^{79}$

Zur Einstimmung auf die AGMB Jahrestagung fand bereits am 15. Mai 2017 an der Universitätsbibliothek der Veterinärmedizinischen Universität Wien das 7. Treffen des AGMBArbeitskreises österreichischer Medizinbibliothekarinnen und -bibliothekare (AGMB.AT) statt. ${ }^{80}$ AGMB.AT versteht sich als Kommunikationsplattform für Personen, die an österreichischen Medizinbibliotheken - an öffentlichen Universitäten, Privatuniversitäten oder Fachhochschulen ${ }^{81}$ tätig sind und an Erfahrungsaustausch und Kooperation interessiert sind.

\subsection{RDA Workshop in Wien 2017}

Am 23. November 2017 war die Universität Wien Veranstalterin des RDA Europe Workshops "From Planning to Action. Towards the Establishment of an Austrian Research Infrastructure", an dem 70 internationale Expertinnen und Experten teilgenommen haben. Im Rahmen der Veranstaltung wurde auch die Gründung einer RDA-Gruppe für Österreich angekündigt. ${ }^{82}$

\footnotetext{
77 Zemanek (2017) und Franke (2017).

78 Blumesberger und Gründhammer (2017).

${ }^{79}$ Frank und Hausberger (2017), Andermann (2017) und Bauer (2018).

${ }^{80}$ Bauer (2017b).

81 Bauer (2018a).

82 Budroni et al. (2017).
} 


\subsection{ODOK 2018}

Von 21. bis 23. Februar 2018 fand an der Universität Wien erstmals eine InetBib-ODOK-Tagung (17. Österreichisches Online-Informationstreffen \&14. InetBib-Tagung) zum Thema „Informationsqualität“ statt, die erstmals gemeinsam von InetBib und VÖB ausgerichtet worden war.

\subsection{Open Access Tage in Graz 2018}

Die Technische Universität Graz war von 24. bis 26. September 2018 Gastgeberin der 12. OpenAccess-Tage, die von 300 Expertinnen und Experten auf dem Gebiet von Open Access besucht wurde. Das Tagungsprogramm wurde dem Motto der "Vielfalt von Open Access" gerecht und erstreckte sich von Open Educational Resources bis zu Open Science. ${ }^{83}$

\subsection{ORCID Austria Workshop 2018}

Am 24. Mai 2018 fand an der Universität für Bodenkultur Wien der ORCID Austria Workshop statt, in dessen Rahmen Vertreterinnen und Vertreter österreichischer Institutionen über ORCID IDs, internationale Best-Practice-Beispiele und die Vorteile einer institutionellen ORCID-Mitgliedschaft informiert haben. ${ }^{84}$

\subsection{SKS-Workshop zu „Focus on Open Science“ in Wien 2018}

Den Themen „FAIR Date and Open Access" und „Open Science and the management of a cultural change" war ein Workshop gewidmet, der am 16. November 2018 von Scientific Knowledge Services (SKS) an der Universität der Technischen Universität Wien ein Workshop zum Thema „Focus on Open Science" (Chapter X: Vienna) veranstaltet wurde.

\subsection{Präsentation der European Open Science Cloud in Wien 2018}

Am 23. November 2018 wurde an der Universität Wien im Rahmen der österreichischen EURatspräsidentschaft die erste Version des Internet-Portals der „European Open Science Cloud“ (EOSC) präsentiert. Dabei sprachen Vertreterinnen und Vertreter aus Politik, Wissenschaft und Forschungsförderung sowie der Europäischen Kommission über Konzept und Bedeutung der EOSC und stellten an konkreten Beispielen deren Potential vor. Im Rahmen dieser Veranstaltung wurde vom österreichischen Bundesminister für Bildung, Wissenschaft und Forschung Heinz Faßmann auch "The Vienna Declaration on the European Open Science Cloud" vorgestellt. ${ }^{85}$

\subsection{1Österreich liest. Treffpunkt Bibliothek 2017 und 2018}

Auch in den letzten beiden Jahren fand die vom Büchereiverband Österreichs (BVÖ) 2006 initiierte und hauptverantwortlich durchgeführte Veranstaltungsreihe „Österreich liest. Treffpunkt Bibliothek" statt, in deren Rahmen von zahlreichen Bibliotheken in Österreich zu einer Fülle an Lesungen, Literaturwanderungen, Bilderbuchkinos, Lesenächten, Literatur-Cafés, Buchausstellungen und vielen

\footnotetext{
83 Pinnitsch und Pichler (2018).

84 Heindl et al. (2018).

85 Bauer (2018c).
} 
weiteren Aktivitäten, die Lust aufs Lesen machen, eingeladen worden ist (16. bis 22. Oktober 2017 bzw. 15. bis 21. Oktober 2018).

\section{Bibliothekspolitik}

\subsection{Publikationen der VÖB}

2017 und 2018 erschienen jeweils drei Ausgaben der von der VÖB herausgegebenen Zeitschrift „Mitteilungen der Vereinigung Österreichischer Bibliothekarinnen und Bibliothekare", darunter insgesamt drei Schwerpunktausgaben zu den Themen "Metadaten - Metadaten", 86 "Repositorien in Österreich - Status quo und (zukünftige) Herausforderungen“87 sowie „ALMA im Österreichischen Bibliothekenverbund“. ${ }^{88}$

Sämtliche Beiträge der "Mitteilungen" werden laufend in bedeutenden Suchmaschinen, Repositorien und Datenbanken indexiert, darunter BASE, DOAJ, DABI Datenbank Deutsches Bibliothekswesen, ELIS, LISTA, ProQuest ${ }^{\circledR}$ Library Science und Scopus.

\subsection{Prekäre Arbeitsverhältnisse im österreichischen Bibliothekswesen}

2015 wurde vom Präsidium der Vereinigung Österreichischer Bibliothekarinnen und Bibliothekare eine Arbeitsgruppe „Prekäre Arbeitsverhältnisse im Bibliothekswesen“ eingerichtet, um die Arbeitssituation von Bibliothekarinnen und Bibliothekaren in Österreich umfassend zu erheben. Von der Arbeitsgruppe wurde eine statistische Erhebung zum bibliothekarischen Arbeitsmarkt mit der Zielsetzung vorbereitet, Informationen zu atypischen Beschäftigungen (Geringfügigkeit, Teilzeit, Befristung) und damit einhergehenden Gefahren der Prekarisierung zu gewinnen.

Im Mai 2017 wurde vom Markt- und Meinungsforschungsinstitut Gallup, das von der VÖB beauftragt wurde, eine Online-Umfrage durchgeführt. 616 Bibliothekarinnen und Bibliothekare beantworteten die 47 Fragen zu ihrer Ausbildung, ihrer bisherigen Laufbahn im Bibliothekswesen, ihrer aktuellen beruflichen Situation sowie zu ihren beruflichen Zukunftsvorstellungen. Die Ergebnisse der Studie wurden 2018 in den „Mitteilungen der Vereinigung Österreichischer Bibliothekarinnen \& Bibliothekare" veröffentlicht. ${ }^{89}$

\subsection{Pressearbeit der VÖB}

Auch 2017 und 2018 wurden die Dienste von APA OTS, einem Dienstleister zur Verbreitung von multimedialen Presse- und Marketinginformationen an Medien, Meinungsbildner und Pressestellen im In- und Ausland, von der VÖB für Pressemeldungen zum österreichischen Bibliothekswesen

\footnotetext{
86 Blumesberger et al. (2017).

87 Blumesberger et al. (2018).

88 Bauer et al. (2018a).

${ }^{89}$ Wödl (2018).
} 
genutzt. Themen waren der 33. Österreichischen Bibliothekartag in Linz, ${ }^{90}$ zur Ausbildungs-Initiative der VÖB mit den Universitätsbibliotheken Wien, Graz und Innsbruck und der OBVSG ${ }^{91}$ sowie eine gemeinsame Erklärung von VÖB und BVÖ zum Bibliotheksgesetz. ${ }^{92}$

\subsection{Kooperation der deutschsprachigen Bibliotheksverbände}

Am 22. und 23. März 2017 fand auf Einladung von Gerald Leitner, dem Generalsekretär der IFLA, ein Treffen von Vertreterinnen und Vertretern des Deutschen Bibliotheksverbands (DBV), des Verbands Bibliothek Information Schweiz (BIS), des Büchereiverbands Österreich (BVÖ) und der Vereinigung Österreichischer Bibliothekarinnen und Bibliothekare (VÖB) im IFLA-Generalsekretariat in der Königlichen Bibliothek in Den Haag statt. Mit dem Treffen sollte abgestimmt werden, wie sich die Verbände des deutschen Sprachraums beim Lobbying auf internationaler Ebene in ihren Aktivitäten besser abstimmen können. Konkrete bibliothekarische Anliegen, die auf politischer Ebene Gehör finden sollten, sind etwa die Schaffung adäquater (Ausnahme-)Regelungen im Urheberrecht bzw. Copyright. Vereinbart wurde, dass das Treffen eine Fortsetzung auf D-A-CH-Ebene finden soll. ${ }^{93}$

\subsection{BAM Austria}

In den vergangenen zwei Jahren wurden von BAM Austria: Arbeitskreis Bibliotheken, Archive, Museen Österreichs zwei Veranstaltungen durchgeführt. ${ }^{94}$

„Metadatennormierung heute für morgen: Open Access und Linked Open Data“ lautete das Thema eines Seminars, das am 11. Mai 2017 in Wien stattgefunden hat und zu dem Vertreterinnen und Vertreter von Bibliotheken, Archiven und Museen eingeladen wurden. Neben Präsentationen, in denen der Mehrwert von Linked Open Data (LOD) thematisiert wurde, standen Best PracticeAnwendungen im Fokus der Veranstaltung.

Im Rahmen des 33. Österreichischen Bibliothekartages in Linz wurde am 13. September 2017 ein Panel zum Thema "Digitalisierung an Bibliotheken, Archiven und Museen" organisiert. Der Präsentation von aktuellen Digitalisierungsinitiativen sowie geplanten zukünftigen Projekten an der Albertina, an der Österreichischen Nationalbibliothek und am Österreichischen Staatsarchiv folgte ein Podiumsgespräch zum Thema „Digitalisierung - wozu?“, das einen Einblick in die Gemeinsamkeiten und Unterschiede der Digitalisierungsstrategien im Bereich des kulturellen Erbes durch die verschiedenen Typen von BAM-Einrichtungen offenbarte.

\footnotetext{
${ }^{90}$ APA OTS (2017a).

${ }^{91}$ APA OTS (2017b).

92 APA OTS (2018).

${ }^{93}$ Ramminger et al. (2017).

${ }^{94}$ Bauer et al. (2017).
} 


\subsection{Memory of Austria}

Die vom österreichischen Memory of the World-Nationalkomitee seit 2014 kuratierte Liste besonders relevanter und typischer Objekte des österreichischen Dokumentenerbes, in der Dokumente und Sammlungen aus Bibliotheken, Archiven, Museen und audiovisuellen Archiven verzeichnet sind, umfasst nach den am 14. September 2018 erfolgten 18 Neuaufnahmen insgesamt 59 Einträge. Neu aufgenommen in das Register wurden für Österreich historisch und kulturell bedeutsame Dokumente und Sammlungen, die folgenden Institutionen zuzuordnen sind: Albertina in Wien und Wienbibliothek im Rathaus (Adolf-Loos-Archiv und Nachlass), Erzabtei St. Peter in Salzburg (Archivbestand des Abtes Dominikus Hagenauer), Österreichisches Staatsarchiv (Confessio Augustana, 1530), Zisterzienserstift Zwettl (Beschreibung der indigenen Bevölkerung in Paraguay, 1770), Diözesanarchiv und -bibliothek Graz-Seckau (Brenner-Bibliothek), Zisterzienserstift Lilienfeld (Concordantiae Caritatis, um 1355), Technisches Museum Wien (Die Fotografien des Oberingenieurs Albert Stächelin zum Bau der Wiener Stadtbahn, 1894-1897), Österreichische Nationalbibliothek und Kunsthistorisches Museum Wien (Die Handschriften der Ambraser Sammlung), Kärntner Landesarchiv (Klagenfurter (oder Ratschacher) Handschrift), Österreichisches Staatsarchiv (Moskauer Memorandum, 1955), Stadtgemeinde Bad Ischl (Nachlass Franz Lehar), Vorarlberger Landesbibliothek (Nachlass von Franz Michael Felder), Österreichische Mediathek (Oral History Interviews mit Opfern des Nationalsozialismus aus Österreich - Sammlung Albert Lichtblau; Sammlung Audio- und Videomitschnitte des Burgtheaters), Wienbibliothek im Rathaus (StraussSammlung), Phonogrammarchiv der Österreichischen Akademie der Wissenschaften (Tonaufnahmen österreichischer Dialekte 1951-1983), Augustiner Chorherrrenstift Vorau (Vorauer Volksbibel, 1467), Österreichisches Staatsarchiv und Österreichische Mediathek (Wissenschaftliche Kommission des Theodor-Körner-Stiftungsfonds und des Leopold-Kunschak-Preises zur Erforschung der österreichischen Geschichte der Jahre 1927-1938)..$^{95}$

Das Internationale Memory of the World- Register, das von der UNESCO seit 1992 betrieben wird, um kulturgeschichtlich einzigartige Dokumente, die exemplarisch das kollektive Gedächtnis der Menschheit repräsentieren, zu würdigen, umfasst unter 427 Eintragungen auch 15 österreichische Dokumente. Am 31. Oktober 2017 erfolgten die Neuaufnahmen des philosophischen Nachlasses von Ludwig Wittgenstein, der an der Österreichischen Nationalbibliothek aufbewahrt wird, und des Dokumentenbestands zur Semmeringbahn, der sich im Bestand des Technischen Museums Wien befindet. ${ }^{96}$

\footnotetext{
95 Pauser (2018).

96 Pauser (2017a).
} 


\subsection{Zukunft Digitalisierung}

Am 7. Juni 2018 wurden von Heinz Faßmann, dem österreichischen Bundesminister für Bildung, Wissenschaft und Forschung, unter dem Motto „Zukunft Digitalisierung - Die österreichischen Universitäten am Weg ins digitale Zeitalter" in einem "Vortrag an den Ministerrat" auch die Entwicklungen an den wissenschaftlichen Bibliotheken angesprochen. Unter den Netzwerken, die bei der Implementierung von Open Access, aber auch bei der Festlegung gemeinsamer Strategien und Vorgehensweisen von großer Bedeutung sind, wurden neben der Österreichischen Universitätenkonferenz (uniko) das Forum Universitätsbibliotheken Österreichs (ubifo), die Österreichische Bibliothekenverbund und Service Gesellschaft m.b.H. (OBVSG), die Kooperation EMedien Österreich (KEMÖ) und das Open Access Network Austria (OANA) angeführt. Hingewiesen wurde auch auf die Bedeutung der Hochschulraum-Strukturmittelprojekte (HRSM), „E-Infrastructures Austria", „E-Infrastructures Austria Plus" und „Austrian Transition to Open Access (AT2OA)“. Für die Zukunft angekündigt wird in Anlehnung an die bisherigen HRSM-Ausschreibungen eine spezielle Ausschreibung zu kooperativen Digitalisierungsschritten der Universitäten. Unter anderem sollen die Inhalte der bestehenden Repositorien in Österreich über ein „Portal Austria“ auffindbar gemacht werden, welches als maßgeblicher Beitrag Österreichs zur „European Open Science Cloud“ zu sehen ist. $^{97}$

\section{Literaturverzeichnis}

Alker-Windbichler, Stefan; Bauer, Bruno (2017): Treuhänderische Übernahme und Verwahrung international und interdisziplinär betrachtet: Tagung zur NS-Provenienzforschung an der Universität Wien (Wien, 2.-4. Mai 2017). In: Mitteilungen der Vereinigung Österreichischer Bibliothekarinnen \& Bibliothekare, 70 (2) 202-13. DOI: https://doi.org/10.31263/voebm.v70i2.1902.

Alker-Windbichler, Stefan; Bauer, Bruno; Stumpf, Markus (2017): NS-Provenienzforschung und Restitution an Bibliotheken. Berlin ; Boston: De Gruyter Saur (Praxiswissen).

Alscher, Hans Joachim (2017): Die Niederösterreichische Landesbibliothek. In: Bibliothek Forschung und Praxis, 41 (1) 89-99. DOI https://doi.org/10.1515/bfp-2016-0006.

Andermann, Heike (2017): „Medizinbibliotheken: inter:disziplinär - inter:national - inter:aktiv“. Jahrestagung der Arbeitsgemeinschaft für Medizinisches Bibliothekswesen (AGMB) e.V. vom 25. bis 27. September 2017 in Wien. In: GMS Medizin Bibliothek Information, 17 (3) Doc22. Verfügbar unter https://dx.doi.org/10.3205/mbi000401.

Andrae, Magdalena; Villányi, Márton (2017): Der Springer Compact-Deal - Ein erster Einblick in die Evaluierung einer Offsetting-Vereinbarung . In: Mitteilungen der Vereinigung Österreichischer Bibliothekarinnen \& Bibliothekare, 70 (2) 274-80. DOI: https://doi.org/10.31263/voebm.v70i2.189.

APA OTS (2017a): VÖB lädt zum 33. Österreichischen Bibliothekartag nach Linz! In: APA OTS, OTS0106. Verfügbar unter https://www.ots.at/presseaussendung/OTS 20170725 OTS0106/voeb-laedt-zum-33oesterreichischen-bibliothekartag-nach-linz.

\footnotetext{
97 Faßmann (2018).
} 
APA OTS (2017b): VÖB sichert berufliche Weiterbildung an wissenschaftlichen Bibliotheken in Österreich. In: APA OTS, OTS0153. Verfügbar unter https://www.ots.at/presseaussendung/OTS 20171207 OTS0153/voeb-sichert-beruflicheweiterbildung-an-wissenschaftlichen-bibliotheken-in-oesterreich.

APA OTS (2018): Gemeinsame Erklärung von VÖB und BVÖ zum Bibliotheksgesetz. In: APA OTS, OTS0165. Verfügbar unter

https://www.ots.at/presseaussendung/OTS 20180529 OTS0165/gemeinsame-erklaerung-vonvoebund-bvoe-zum-bibliotheksgesetz.

Bauer, Bruno (2017a): „Austrian Transition to Open Access“ 2017-2020. In: GMS Medizin Bibliothek Information, 17 (3) Doc15. Verfügbar unter https://dx.doi.org/10.3205/mbi000394.

Bauer, Bruno (2017b): 7. Treffen des AGMB-Arbeitskreises österreichischer Medizinbibliothekarinnen und -bibliothekare (AGMB.AT) an der Vetmeduni Wien am 15. Mai 2017. In: GMS Medizin Bibliothek Information, 17 (1-2) Doc03. Verfügbar unter https://dx.doi.org/10.3205/mbi000382.

Bauer, Bruno (2017c): Open Access In Österreich (4a). In: Söllner, Konstanze; Mittermaier, Bernhard (Hrsg.): Praxishandbuch Open Access. Berlin, Boston: de Gruyter, 207-14. (De Gruyter Praxishandbuch).

Bauer, Bruno (2017d): Qualitätsmanagement und Zertifizierung: Aufwand und Nutzen am Beispiel der Universitätsbibliothek der Medizinischen Universität Wien. In: BIBLIOTHEK - Forschung und Praxis, 41 (3) 305-18. Verfügbar unter https://doi.org/10.1515/9783110522334.

Bauer, Bruno (2018a): Kooperationen bei der Literatur- und Informationsversorgung von medizinischen Fakultäten und Hochschulen in Österreich. In: GMS Medizin Bibliothek Information, 18 (1-2) Doc07. Verfügbar unter doi: https://dx.doi.org/10.3205/mbi000402.

Bauer, Bruno (2018b): Medizinbibliotheken: inter:disziplinär - inter:national - inter:aktiv. Jahrestagung der Arbeitsgemeinschaft für Medizinisches Bibliothekswesen (AGMB) vom 25. bis 27. September 2017 in Wien. In: ZfBB, 65 (2-3) 125-29. DOI: http://dx.doi.org/10.3196/18642950186523154.

Bauer, Bruno (2018c): Präsentation der European Open Science Cloud an der Universität Wien (Wien, 23. November 2018). In: Mitteilungen der Vereinigung Österreichischer Bibliothekarinnen \& Bibliothekare, 71 (3/4) 524-29. DOI: https://doi.org/10.31263/voebm.v71i3-4.2171.

Bauer, Bruno (2018d): Sieben Jahre kontinuierlicher Verbesserungsprozess gemäß DIN EN ISO 9001: Ein Erfahrungsbericht über die erfolgreiche Nutzung dieses Qualitätsmanagementsystems an der Universitätsbibliothek der Medizinischen Universität Wien. In: GMS Medizin Bibliothek Information, 18 (3) Doc15. Verfügbar unter doi: https://dx.doi.org/10.3205/mbi000416.

Bauer, Bruno; Capellaro, Christof; Ferus, Andreas; Fessler, Georg; Granat, Renata; Hölbling, Lothar; Kaier, Christian; Koren-Wilhelmer, Frank; Kromp, Brigitte; Seissl, Maria; Zarka, Tobias (2018): Austrian Transition to Open Access (AT2OA). In: BIBLIOTHEK - Forschung und Praxis, 42 (3)46375. DOI: https://doi.org/10.1515/bfp-2018-0062.

Bauer, Bruno; Budroni, Paolo; Ferus, Andreas; Ganguly, Raman; Ramminger, Eva; Sánchez Solís, Barbara (2017): e-Infrastructures Austria 2016: Bericht über das dritte Jahr des Hochschulraumstrukturmittelprojekts für den koordinierten Aufbau und die kooperative Weiterentwicklung von Repositorieninfrastrukturen. In: Mitteilungen der Vereinigung Österreichischer Bibliothekarinnen \& Bibliothekare, 70 (1) 66-93. DOI: https://doi.org/10.31263/voebm.v70i1.1834.

Bauer, Bruno; Feigl, Markus; Fröschl, Gabriele; Griesser, Martina; Hubert, Rainer; Mikoletzky, Lorenz; Schmidt, Alfred; Wendelin, Harald (2017). BAM Austria: Arbeitskreis Bibliotheken, Archive, Museen Österreichs - Aktivitäten 2017. In: Mitteilungen der Vereinigung Österreichischer Bibliothekarinnen \& Bibliothekare, 70 (3/4) 350-56. DOI: https://doi.org/10.31263/voebm.v70i3.1957.

Bauer, Bruno; Ferus, Andreas (2018): Österreichische Repositorien in OpenDOAR und re3data.org: Entwicklung und Status von Infrastrukturen für Green Open Access und Forschungsdaten. In: 
Mitteilungen der Vereinigung Österreichischer Bibliothekarinnen \& Bibliothekare, 71 (1) 70-86. DOI: https://doi.org/10.31263/voebm.v71i1.2037.

Bauer, Bruno; Hamedinger, Wolfgang (2018): Alma im Österreichischen Bibliothekenverbund und die Perspektiven für die Österreichische Bibliothekenverbund und Service GmbH. 10 Fragen von Bruno Bauer an Wolfgang Hamedinger, Geschäftsführer der OBVSG. In: Mitteilungen der Vereinigung Österreichischer Bibliothekarinnen \& Bibliothekare, 71 (2), 356-371. DOI: https://doi.org/10.31263/voebm.v71i2.2138.

Bauer, Bruno; Lackner, Markus; Schubert, Bernhard (2018a): Der Österreichische Bibliothekenverbund im Umbruch: Neues Bibliotheksverwaltungssystem Alma als Impuls für die Zukunft. Editorial. In: Mitteilungen der Vereinigung Österreichischer Bibliothekarinnen \& Bibliothekare, 71 (2), 260-266. DOI: https://doi.org/10.31263/voebm.v71i2.2130.

Bauer, Bruno; Lackner, Markus; Schubert, Bernhard (2018b): Implementierung des neuen Bibliotheksmanagementsystems Alma an 14 Einrichtungen im Österreichischen Bibliothekenverbund - Feedback aus der Perspektive der Functional Experts. In: Mitteilungen der Vereinigung Österreichischer Bibliothekarinnen \& Bibliothekare, 71 (2), 320-350. DOI: https://doi.org/10.31263/voebm.v71i2.2136.

Baumgartner, Edwin (2017): Klub der Geheimniskrämer. In: Wiener Zeitung. Verfügbar unter https://www.wienerzeitung.at/nachrichten/kultur/kunst/900638_Klub-derGeheimniskraemer.html, veröffentlicht am 26.06.2017.

Beiler, Christian; Gratzl, Petra; Schubert, Bernhard; Steiner, Christoph; Steltzer, Rainer (2018): Erschließungsarbeit in Alma - Erfahrungen aus dem OBV vor, während und nach der AlephAblöse. In: Mitteilungen der Vereinigung Österreichischer Bibliothekarinnen \& Bibliothekare, 71 (2), 282-306. DOI: https://doi.org/10.31263/voebm.v71i2.2134.

Bittermann-Wille, Christa; Jammernegg, Lydia (2017): Das frauen- und geschlechterspezifische Gedächtnis einer Universalbibliothek. In: Bibliothek Forschung und Praxis, 41 (2), S. 140-148. DOI: https://doi.org/10.1515/bfp-2017-0029.

Blumesberger, Susanne (2017): Herausforderungen für Repositorien!?“ Tagung anlässlich 10 Jahre Phaidra an der Universität Wien (Wien, 24. Oktober 2017). In: Mitteilungen der Vereinigung Österreichischer Bibliothekarinnen \& Bibliothekare, 70 (3/4), 372-376. DOI: https://doi.org/10.31263/voebm.v70i3.1960.

Blumesberger, Susanne (2018): Neue Anforderungen - viele offene Fragen. Zu den vielfältigen Rollen von Repositorien am Beispiel der UB Wien. In: Mitteilungen der Vereinigung Österreichischer Bibliothekarinnen \& Bibliothekare, 71 (1), 149-161. DOI: https://doi.org/10.31263/voebm.v71i1.2003.

Blumesberger, Susanne; Ferus, Andreas; Kaier, Christian; Novotny, Gertraud (2018): Schwerpunktthema „Repositorien in Österreich - Status quo und (zukünftige) Herausforderungen“. Editorial. In: Mitteilungen der Vereinigung Österreichischer Bibliothekarinnen \& Bibliothekare, 71 (1), 5-7. DOI: https://doi.org/10.31263/voebm.v71i1.2041.

Blumesberger, Susanne; Gründhammer, Veronika (2017): Digitale Bibliothek. Zukunft des digitalen Erbes? Nachhaltige Lösungen für Gedächtnis- und Wissenschaftseinrichtungen (Graz, 2.-3. März 2017). In: Mitteilungen der Vereinigung Österreichischer Bibliothekarinnen \& Bibliothekare, 70 (1), 128-133. DOI: https://doi.org/10.31263/voebm.v70i1.1841.

Blumesberger, Susanne; Schubert, Bernhard; Zartl, Alexander (2017): Schwerpunktthema „Metadata - Metadaten“. Editorial. In: Mitteilungen der Vereinigung Österreichischer Bibliothekarinnen \& Bibliothekare, 70 (2), 153-156. DOI: https://doi.org/10.31263/voebm.v70i2.1851.

Blumesberger, Susanne; Zartl, Alexander (2017): Umgang mit Metadaten in Repositorien - Eine österreichweite Umfrage. In: Mitteilungen der Vereinigung Österreichischer Bibliothekarinnen \& Bibliothekare, 70 (2), 249-273. DOI: https://doi.org/10.31263/voebm.v70i2.1850. 
Brantl, Vera (2018): Berta Zuckerkandl - Netzwerkerin der Wiener Moderne: Über die Sammlungen Emile Zuckerkandl an der Österreichischen Nationalbibliothek. In: Bibliothek Forschung und Praxis, 42 (1), S. 128-135. DOI: https://doi.org/10.1515/bfp-2018-0017.

Budroni, Paolo; Ganguly, Raman; Miksa, Tomasz; Rauber, Andreas; Sánchez Solís, Barbara (2017): RDA Europe Workshop - From Planning to Action. Towards the Establishment of an Austrian Research Infrastructure (Wien, 23. November 2017). In: Mitteilungen der Vereinigung Österreichischer Bibliothekarinnen \& Bibliothekare, 70 (3/4), 382-389. DOI: https://doi.org/10.31263/voebm.v70i3.1962.

Buland, Rainer; Leitner, Edith (2018): „Zeige mir alle Bilder, auf denen Würfel zu sehen sind!“ Die Veröffentlichung der Graphiken des Instituts für Spielforschung im Repositorium der UB Mozarteum. In: Mitteilungen der Vereinigung Österreichischer Bibliothekarinnen \& Bibliothekare, 71 (1), 30-40. DOI: https://doi.org/10.31263/voebm.v71i1.1988.

Bundeskanzleramt/Bundesregierung/Nachrichten (2018): Kulturminister Blümel eröffnete Ausstellung „Schatzkammer des Wissens - 650 Jahre Österreichische Nationalbibliothek". Verfügbar unter https://www.bundeskanzleramt.gv.at/-/kulturminister-blumel-eroffneteausstellung-schatzkammer-des-wissens-650-jahre-osterreichische-nationalbibliothek-, veröffentlicht am 25.01.2018.

Eberhard, Igor; Kraus, Wolfgang (2018): Der Elefant im Raum. Ethnographisches Forschungsdatenmanagement als Herausforderung für Repositorien. In: Mitteilungen der Vereinigung Österreichischer Bibliothekarinnen \& Bibliothekare, 71 (1), 41-52. DOI: https://doi.org/10.31263/voebm.v71i1.2018.

Eberle, Harald; Feurstein, Thomas (2018): volare - das Vorarlberger Landesrepositorium. In: Mitteilungen der Vereinigung Österreichischer Bibliothekarinnen \& Bibliothekare, 71 (3/4), 407415. DOI: https://doi.org/10.31263/voebm.v71i3-4.2161.

Faßmann, Heinz (2018): Vorhaben des Bundesministers für Bildung, Wissenschaft und Forschung zur Zukunft Digitalisierung - Die österreichischen Universitäten am Weg ins digitale Zeitalter. Vortrag an den Ministerrat. Verfügbar unter https://www.bundeskanzleramt.gv.at/documents/131008/877075/21_9_mrv.pdf/1e321eea066c-4d71-8143-b757d21cfe21, veröffentlicht am 07.06.2018.

Festakt zum 650-Jahr-Jubiläum der Nationalbibliothek (2018). In: Salzburger Nachrichten. Verfügbar unter https://www.sn.at/kultur/allgemein/festakt-zum-650-jahr-jubilaeum-dernationalbibliothek-24561961, veröffentlicht am 22.02.2018.

Frank, David; Hausberger, Claudia (2017): Medizinbibliotheken: Inter:disziplinär - Inter:national Inter:aktiv an der Veterinär- medizinischen Universität Wien (Wien, 25.-27. September 2017). In: Mitteilungen der Vereinigung Österreichischer Bibliothekarinnen \& Bibliothekare, 70 (3/4), 364371. DOI: https://doi.org/10.31263/voebm.v70i3.1959.

Franke, Fabian (2017): 1. Informationskompetenz-Tag Deutschland/Österreich am 16./17.02.2017 in Innsbruck. In: o-bib. Das offene Bibliotheksjournal, 4 (2), S. 101-104. Verfügbar unter doi: https://doi.org/10.5282/o-bib/2017H2S101-104.

Franke, Fabian; Söllner, Konstanze; Syré, Ludger (2018): „Wolkenkuckucksheim. Bibliotheken in der Cloud". Österreichischer Bibliothekartag vom 12. bis 15. September 2017 in Linz. In: ABI Technik, 38 (1), S. 85-95. DOI: https://doi.org/10.1515/abitech-2018-0012.

Hagmann, Dominik (2018). Überlegungen zur Nutzung von PHAIDRA als Repositorium für digitale archäologische Daten. In: Mitteilungen der Vereinigung Österreichischer Bibliothekarinnen \& Bibliothekare, 71 (1), 53-69. DOI: https://doi.org/10.31263/voebm.v71i1.1974.

Heider, Veronika (2018): AUSSDA - Das sozialwissenschaftliche Datenarchiv mit europäischer Komponente. In: Mitteilungen der Vereinigung Österreichischer Bibliothekarinnen \& Bibliothekare, 71 (1), 181-189. DOI: https://doi.org/10.31263/voebm.v71i1.1996. 
Heindl, Markus; Hikl, Anna-Laetitia; Kaier, Christian (2018): ORCID Austria Workshop (Wien, 24. Mai 2018). In: Mitteilungen der Vereinigung Österreichischer Bibliothekarinnen \& Bibliothekare, 71 (3/4), 468-474. DOI: https://doi.org/10.31263/voebm.v71i3-4.2109.

Jandl, Paul (2018): Neue Dokumente öffnen den Blick in das Universum der jungen Ingeborg Bachmann. Neue Zürcher Zeitung, 27.06.2018. Online: https://www.nzz.ch/feuilleton/neuedokumente-oeffnen-den-blick-in-das-universum-der-ingeborg-bachmann-ld.1398213.

Kaier, Christian; Lackner, Karin (2018): Publikationsunterstützung an der Universität Graz: Themen, Zielgruppen, Formate. In: Bibliothek Forschung und Praxis, 42 (1), S.116-121. DOI: https://doi.org/10.1515/bfp-2018-0011.

Kaier, Christian; Schilhan, Lisa (2018): Gold-Open-Access-Zeitschriften auf dem institutionellen Repositorium unipub. In: Mitteilungen der Vereinigung Österreichischer Bibliothekarinnen \& Bibliothekare, 71 (1), 190-198. DOI: https://doi.org/10.31263/voebm.v71i1.1975.

Kaiser, Olivia; Köstner, Christina; Stumpf, Markus (Hrsg.)(2017): Treuhänderische Übernahme und Verwahrung : international und interdisziplinär betrachtet. Tagung "Treuhänderische" Übernahme und Verwahrung - International und Interdisziplinär Betrachtet, Wien: V \& R unipress GmbH. DOI: 10.14220/9783737007832.

Kann, Bettina (2017): Alma im Österreichischen Bibliothekenverbund: Werkstattbericht. In: Bibliotheksdienst, 51 (7), 562-574. DOI: 10.1515/bd-2017-0061.

Kann, Bettina (2018): Alma im Österreichischen Bibliothekenverbund (OBV): Aus der Werkstatt der OBVSG. In: Mitteilungen der Vereinigung Österreichischer Bibliothekarinnen \& Bibliothekare, 71 (2), 307-319. DOI: https://doi.org/10.31263/voebm.v71i2.2133.

Kieslinger, Christian; Rathmanner, Karl (2018): Von Alephino nach Alma, ein Dramolett in drei Akten. Ein Erfahrungsbericht der Bibliothek der Fachhochschule Sankt Pölten. In: Mitteilungen der Vereinigung Österreichischer Bibliothekarinnen \& Bibliothekare, 71 (2), 276-281. DOI: https://doi.org/10.31263/voebm.v71i2.2132.

Klien, Peter (2017): Mitteilungen aus der OBVSG. In: Mitteilungen der Vereinigung Österreichischer Bibliothekarinnen \& Bibliothekare, 70 (1), 100-102. DOI: https://doi.org/10.31263/voebm.v70i1.1836.

Knieling, Nina; Huber-Frischeis, Thomas; Valenta, Rainer; Petschar, Hans (2018): Die HabsburgLothringische Familien-Fideikommissbibliothek vom Tod Kaiser Franz I. 1835 bis zu ihrer Eingliederung in die Nationalbibliothek der Republik Österreich 1921. In: Bibliothek Forschung und Praxis, 42 (1), S. 154-161. DOI: https://doi.org/10.1515/bfp-2018-0014.

Kocher-Lichem, Katharina (2017): Die Steiermärkische Landesbibliothek im neuen Joanneumsviertel. In: Bibliothek Forschung und Praxis, 41 (2), S. 167-174. DOI: https://doi.org/10.1515/bfp-20170026.

Koll, Johannes; Zodl, Regina (2017): Provenienzforschung ins Museum: Ausstellungseröffnung und Restitution der Wirtschaftsuniversität Wien im Technischen Museum Wien. In: Mitteilungen der Vereinigung Österreichischer Bibliothekarinnen \& Bibliothekare, 70 (3/4), 342-349. DOI: https://doi.org/10.31263/voebm.v70i3.1956.

Kromp, Brigitte; Mayer, Wolfgang (2017): Shared Archiving Austria - Eine gemeinsame Archivierungsstrategie für österreichische Universitätsbibliotheken. In: ABI Technik, 37 (2), S. 111118. DOI: https://doi.org/10.1515/abitech-2017-0024.

Kronschläger, Mathis (2018a): Mitteilungen der OBVSG. In: Mitteilungen der Vereinigung Österreichischer Bibliothekarinnen \& Bibliothekare, 71 (2), 372-377. DOI: https://doi.org/10.31263/voebm.v71i2.2140.

Kronschläger, Mathis (2018b): Mitteilungen der OBVSG. In: Mitteilungen der Vereinigung Österreichischer Bibliothekarinnen \& Bibliothekare, 71 (3/4), 453-458. DOI: https://doi.org/10.31263/voebm.v71i3-4.2166. 
Kronschläger, Mathis (2018c): Verbundtag 2018 (Klagenfurt, 15. Mai 2018). In: Mitteilungen der Vereinigung Österreichischer Bibliothekarinnen \& Bibliothekare, 71 (2), 378-380. DOI: https://doi.org/10.31263/voebm.v71i2.2141.

Lackner, Karin; Ginther, Clara (2017): Unterstützung des wissenschaftlichen Publizierens als Aufgabe einer wissenschaftlichen Bibliothek: Die Publikationsservices an der UB Graz. In: o-bib. Das offene Bibliotheksjournal, 4 (4), S. 145-154. DOI: https://doi.org/10.5282/o-bib/2017H4S145-154.

Lackner, Markus (2018): Die Alma-Implementierung im Österreichischen Bibliothekenverbund (OBV) unter besonderer Berücksichtigung der Kohorte 2-Bibliotheken. In: Mitteilungen der Vereinigung Österreichischer Bibliothekarinnen \& Bibliothekare, 71 (2), 267-275. DOI: https://doi.org/10.31263/voebm.v71i2.2131.

Lahner, Irmgard (2017): Universitätsbibliothek Salzburg restituierte Bücher und handschriftliche Manuskripte aus dem Konradinum in Eugendorf. In: Mitteilungen der Vereinigung Österreichischer Bibliothekarinnen \& Bibliothekare, 70 (1), 94-99. DOI: https://doi.org/10.31263/voebm.v70i1.1835.

Manojlovic, Katharina (2017): Labor, Speicher, Bühne: Das Literaturmuseum der Österreichischen Nationalbibliothek. In: Bibliothek Forschung und Praxis, 41 (2), S. 175-184. DOI: https://doi.org/10.1515/bfp-2017-0006.

Mayr, Michaela (2018): Vision und Strategieentwicklung der Österreichischen Nationalbibliothek. In: Bibliothek Forschung und Praxis, 42 (1), S. 122-127. DOI: https://doi.org/10.1515/bfp-2018-0016.

Müller, Christa; Mokre, Jan; Hintersonnleitner, Michael (2017): AKON - Ansichtskarten Online. In: Bibliothek Forschung und Praxis, 41 (2), S. 149-155. DOI: https://doi.org/10.1515/bfp-2017-0031.

Nationalbibliothek ersteigert Teilnachlass Ingeborg Bachmanns. 1.200 Seiten mit Notizen und Briefen aus Studienzeit der Autorin für 130.000 Euro erworben (2018). In: Der Standard. Verfügbar unter https://www.derstandard.de/story/2000082198058/nationalbibliothek-ersteigert-teilnachlassingeborg-bachmanns, veröffentlicht am 25.06.2018.

Nationalbibliothek hat Morton-Nachlass und Klüger-Vorlass bekommen (2018). In: Der Standard. Verfügbar unter https://derstandard.at/2000080244905/Nationalbibliothek-bekommt-MortonNachlass-und-Klueger-Vorlass, veröffentlicht am 23.05.2018.

Nowak, Melanie; Schneckenleithner, Clara (2017): „Wohin mit den InformationsexpertInnen?“ Ergebnisse einer Erhebung zur Berufseinstiegssituation der AbsolventInnen des Grundlehrgangs „Library and Information Studies". In: Mitteilungen der Vereinigung Österreichischer Bibliothekarinnen \& Bibliothekare, 70 (1), 42-65. DOI: https://doi.org/10.31263/voebm.v70i1.1833.

OBV (2017a): Alles neu macht ALMA. Auswirkungen des Umstiegs auf PRIMO. In: OBV SG Newsletter. Aktuelles aus dem Österreichischen Bibliothekenverbund (OBV), (2), 2.

OBV (2017b): Come Together Right Now. Verbundtag 2017 an der Universitäts- und Landesbibliothek Tirol. In: OBV SG Newsletter. Aktuelles aus dem Österreichischen Bibliothekenverbund (OBV), (1), 1.

OBV (2017c): Das digitale Schatzkästlein. Die „Bibliotheca Mozartiana digital“. Der Stiftung Mozarteum. In: OBV SG Newsletter. Aktuelles aus dem Österreichischen Bibliothekenverbund (OBV), (1), 3.

OBV (2017d): Retroprojekt für die Arbeiterkammer Wien beendet. In: OBV SG Newsletter. Aktuelles aus dem Österreichischen Bibliothekenverbund (OBV), (2), 2.

OBV (2017e): Retroprojekt für die Wienbibliothek im Rathaus begonnen. In: OBV SG Newsletter. Aktuelles aus dem Österreichischen Bibliothekenverbund (OBV), (2), 2.

OBV (2017f): Schreck, nach lass. Datenbank „Nachlässe, Autographen und Handschriften“ nun Teil der Verbunddatenbank. In: OBV SG Newsletter. Aktuelles aus dem Österreichischen Bibliothekenverbund (OBV), (2), 3. 
OBV (2017g): Visual Library macht (Fachhoch-)Schule. Open Access publizieren mit Visual Library. In: OBV SG Newsletter. Aktuelles aus dem Österreichischen Bibliothekenverbund (OBV), (2), 3.

OBV (2017h): Zukünftige Dienstleistungen der OBVSG? Ergebnisse einer Umfrage unter den Verbundbibliotheken. In: OBV SG Newsletter. Aktuelles aus dem Österreichischen Bibliothekenverbund (OBV), (1), 3.

OBV (2018a): Alles ALMA. Der Go-Live der ALMA Netzwerkzone. In: OBV SG Newsletter. Aktuelles aus dem Österreichischen Bibliothekenverbund (OBV), (1), 2.

OBV (2018b): Gestärkt in die neue Laufzeit. Kooperation E-Medien setzt weiterhin Schwerpunkt auf Open Access. In: OBV SG Newsletter. Aktuelles aus dem Österreichischen Bibliothekenverbund (OBV), (1), 3.

OBV (2018c): Herzlich willkommen! Prisrcno dobrodosli! Benvenuto! Verbundtag 2018 an der Universitätsbibliothek Klagenfurt. In: OBV SG Newsletter. Aktuelles aus dem Österreichischen Bibliothekenverbund (OBV), (1), 1.

OBV (2018d): Primo4you, Primo4me, Primo4Alma. Der Umstieg der Verbundsuchmaschine auf ALMA. In: OBV SG Newsletter. Aktuelles aus dem Österreichischen Bibliothekenverbund (OBV), (1), 3.

OBV (2018e): Retroprojekt für die Wienbibliothek im Rathaus erfolgreich abgeschlossen. In: OBV SG Newsletter. Aktuelles aus dem Österreichischen Bibliothekenverbund (OBV), (2), 2.

ÖNB restituierte 2255 Bücher an den Nationalfonds (2018). In: Salzburger Nachrichten. Verfügbar unter https://www.sn.at/kultur/allgemein/oenb-restituierte-2255-buecher-an-den-nationalfonds62523985, veröffentlicht am 14.12.2019.

ORF übernimmt Patenschaften über drei Bücher zur Geschichte des ORF: ÖNB-Generaldirektorin Rachinger überreicht Urkunden an ORF-Generaldirektor Wrabetz (2017). In: ORF Unternehmen. Verfügbar unter https://der.orf.at/unternehmen/aktuell/buchpatenschaft100.html, veröffentlicht am 15.11.2017.

Ortner, Michaela (2017): Die Katalogisierung der Bücher der ehemaligen Fideikommissbibliothek des Hauses Habsburg- Lothringen an der Österreichischen Nationalbibliothek. In: Bibliothek Forschung und Praxis, 41 (2), S. 156-162. DOI: https://doi.org/10.1515/bfp-2017-0030.

Österreichische Nationalbibliothek Prunksaal: Schatzkammer des Wissens. 650 Jahre Österreichische Nationalbibliothek. Verfügbar unter https://www.onb.ac.at/museen/prunksaal/sonderausstellungen/schatzkammer-des-wissens/.

Pauser, Josef (2017a): Der philosophische Nachlass von Ludwig Wittgenstein und die historischen Dokumente zum Bau der Semmeringbahn ab nun UNESCO-Weltdokumentenerbe. In: VÖBBLOG. Verfügbar unter https://www.univie.ac.at/voeb/blog/?p=44770, veröffentlicht am 31.10.2018.

Pauser, Josef (2017b): Neuer Standort der Ausbildungsabteilung der ÖNB. In: VÖBBLOG. Verfügbar unter https://www.univie.ac.at/voeb/blog/?p=42746, veröffentlicht am 07.03.2017.

Pauser, Josef (2017c): Österreichische Nationalbibliothek erhält Nachlass von Trude Marzik. In: VÖBBLOG. Verfügbar unter http://www.univie.ac.at/voeb/blog/?p=42965, veröffentlicht am 03.03.2017.

Pauser, Josef (2018): UNESCO: 18 Neuaufnahmen in das nationale Memory of the World-Register „Memory of Austria“. In: VÖBBLOG. Verfügbar unter https://www.univie.ac.at/voeb/blog/?p=47098, veröffentlicht am 14.09.2018.

Perschy, Jakob Michael (2018): Die Burgenländische Landesbibliothek - ein offenes Fenster nach Osten. In: Bibliothek Forschung und Praxis, 42 (3), S. 486-488. DOI: https://doi.org/10.1515/bfp2018-0055.

Petritsch, Barbara; Porsche, Jana (2018): IST PubRep und IST DataRep: die institutionellen Repositorien am IST Austria. In: Mitteilungen der Vereinigung Österreichischer Bibliothekarinnen \& Bibliothekare, 71 (1), 199-206. DOI: https://doi.org/10.31263/voebm.v71i1.1993. 
Pinnitsch, Simone; Pichler, Franz (2018): Open-Access-Tage 2018 - „Vielfalt von Open Access“ (Graz, 24.-26. September 2018). Open-Access-Tage 2018 - „Vielfalt von Open Access“ (Graz, 24.-26. September 2018). In: Mitteilungen der Vereinigung Österreichischer Bibliothekarinnen \& Bibliothekare, 71 (3/4), 508-513. DOI: https://doi.org/10.31263/voebm.v71i3-4.2144.

Pum, Gabriele (2018): News aus dem Bereich der bibliothekarischen Ausbildung in Österreich im Jahr 2017 und 2018. In: Mitteilungen der Vereinigung Österreichischer Bibliothekarinnen \& Bibliothekare, 71 (3/4), 425-432. DOI: https://doi.org/10.31263/voebm.v71i3-4.2163.

Rachinger, Johanna (2018): Rede von ÖNB-Direktorin Johanna Rachinger. Die Österreichische Nationalbibliothek (ÖNB) hat am Donnerstag mit einem Festakt ihr 650-Jahr-Jubiläum gefeiert. In: wien.ORF.at. Verfügbar unter https://wien.orf.at/news/stories/2897060/, veröffentlicht am 22.02.2018.

Ramminger, Eva; Andrae, Magdalena; Torggler, Andrea (2017): „Libraries.Solidarity.Society“ Schritte zu einer Intensivierung der Kooperation zwischen den deutschsprachigen und internationalen Bibliotheksverbänden. In: Mitteilungen der Vereinigung Österreichischer Bibliothekarinnen \& Bibliothekare, 70 (2), 284-289.

Reckling, Falk; Rieck, Katharina (2018): 4. Informationsveranstaltung des Open Access Network Austria (OANA) (Wien, 10. Jänner 2018). In: Mitteilungen der Vereinigung Österreichischer Bibliothekarinnen \& Bibliothekare, 71 (3/4), 461-467. DOI: https://doi.org/10.31263/voebm.v71i34.2169 .

Schau zu 300 Jahre Maria Theresia in der Nationalbibliothek (2017). In: Salzburger Nachrichten. Verfügbar unter https://www.sn.at/kultur/schau-zu-300-jahre-maria-theresia-in-dernationalbibliothek-369571, veröffentlicht am 15.02.2017.

Schiller, Robert (2018): Wo gehest Du hin? Zukünftige Dienstleistungen der OBVSG - Ergebnisse einer Umfrage. In: Mitteilungen der Vereinigung Österreichischer Bibliothekarinnen \& Bibliothekare, 71 (2), 351-355. DOI: https://doi.org/10.31263/voebm.v71i2.2137.

Schlacher, Werner (2018): Der Umbau der Universitätsbibliothek Graz - ein Bericht in drei Fortsetzungen - Teil 2: Vom Fall und Aufstieg eines Gebäudes. In: Mitteilungen der Vereinigung Österreichischer Bibliothekarinnen \& Bibliothekare, 71 (3/4), 416-424. DOI: https://doi.org/10.31263/voebm.v71i3-4.2162.

Schmidt, Alfred (2018): Neue Online-Aktivitäten der Österreichischen Nationalbibliothek. In: Bibliothek Forschung und Praxis, 42 (1), S. 136-140. DOI: https://doi.org/10.1515/bfp-2018-0019.

Stigler, Johannes Hubert; Steiner, Elisabeth (2018): GAMS - Eine Infrastruktur zur Langzeitarchivierung und Publikation geisteswissenschaftlicher Forschungsdaten. In: Mitteilungen der Vereinigung Österreichischer Bibliothekarinnen \& Bibliothekare, 71 (1), 207-216. DOI: https://doi.org/10.31263/voebm.v71i1.1992.

Torggler, Andrea; Andrae, Magdalena (2018): Aus dem Leben einer/s Repman - ein Bericht aus dem österreichischen „Netzwerk für RepositorienmanagerInnen“. In: Mitteilungen der Vereinigung Österreichischer Bibliothekarinnen \& Bibliothekare, 71 (1), 107-124. DOI: https://doi.org/10.31263/voebm.v71i1.1986.

Trognitz, Martina; Ďurčo, Matej (2018): Ein Schema, sie alle zu binden. Das Innenleben des digitalen Archivs ARCHE. In: Mitteilungen der Vereinigung Österreichischer Bibliothekarinnen \& Bibliothekare, 71 (1), 217-231. DOI: https://doi.org/10.31263/voebm.v71i1.1979.

Tuider, Bernhard (2017): Die Sammlung für Plansprachen und das Esperantomuseum der Österreichischen Nationalbibliothek - Geschichte, Bestand und Projekte. In: Bibliothek Forschung und Praxis, 41 (2), S. 185-192. DOI: https://doi.org/10.1515/bfp-2017-0027.

Van der Bellen, Alexander (2018): „Nicht zufällig sind Bibliotheken seit jeher wahre Sehnsuchtsorte“. Rede von Bundespräsident Alexander Van der Bellen beim Festakt zum 650-jährigen Bestehen der Österreichischen Nationalbibliothek. Verfügbar unter https://bundespraesident.at/aktuelles/detail/news/nicht-zufaellig-sind-bibliotheken-seit-jeherwahre-sehnsuchtsorte/, veröffentlicht am 22.02.2018. 
Villányi, Márton (2018): Lizenzverträge mit Open-Access-Komponenten an österreichischen Bibliotheken. Master-Thesis (ULG), Universität Wien. Universitätslehrgang Library and Information Studies (MSc). Verfügbar unter http://othes.univie.ac.at/51113/.

Wiese, Silvio (2018): Die OBVSG, Visual Library und eine stetig wachsende Anzahl von Publikationen und NutzerInnen. In: Mitteilungen der Vereinigung Österreichischer Bibliothekarinnen \& Bibliothekare, 71 (1), 232-238. DOI: https://doi.org/10.31263/voebm.v71i1.1978.

Wiley, Kooperation E-Medien Österreich und FWF geben kombinierte Open-Access Publikations- und Subskriptions-Vereinbarung bekannt (2018). Wien: KEMÖ Meldung, Nr. 848. Verfügbar unter https://www.konsortien.at/meldungen-Details.asp?meldungenid=848, veröffentlicht am 12.02.2018.

Wödl, Ute (2018): Prekäre Arbeitsverhältnisse im Bibliothekswesen. In: Mitteilungen der Vereinigung Österreichischer Bibliothekarinnen \& Bibliothekare, 71 (3/4), 433-450. DOI: https://doi.org/10.31263/voebm.v71i3-4.2164.

Wurmitzer, Michael (2018): 650 Jahre Nationalbibliothek: Zeitreise durch die Identität eines Landes. In: Der Standard. Verfügbar unter https://derstandard.at/2000073013431/650-Jahre-OeNBZeitreise-durch-die-Identitaet-eines-Landes, veröffentlicht am 26.01.2018.

Zauner, Thomas (2017): Maria Theresia sagt: Danke! In: Bibliothek Forschung und Praxis, 41 (2), S. 163-166. DOI: https://doi.org/10.1515/bfp-2017-0028.

Zemanek, Michaela (2017): 1. Informationskompetenz-Tagung Deutschland / Österreich (Innsbruck, 16.-17. Februar 2017). In: Mitteilungen der Vereinigung Österreichischer Bibliothekarinnen \& Bibliothekare, 70 (1), 111-114. DOI: https://doi.org/10.31263/voebm.v70i1.1839.

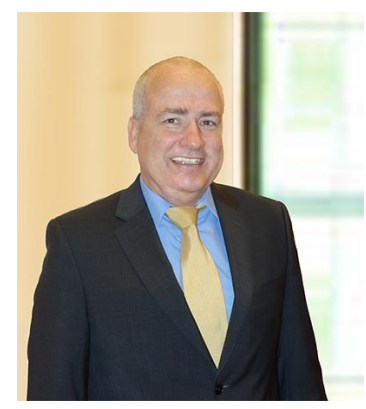

Mag. Bruno Bauer

Leiter der Universitätsbibliothek

Medizinische Universität Wien

Währinger Gürtel 18-20

A-1097 Wien

Österreich

bruno.bauer@meduniwien.ac.at

orcid.org/0000-0002-4729-331X 